\title{
Simulation probabiliste de l'usure dans les contacts roulants sous charges
}

\author{
Sylvain Cloupet $^{1}$, Luc Chevalier ${ }^{2, a}$ et Hugues Chollet $^{1}$ \\ ${ }^{1}$ LTN - INRETS, 2 Av. du Général Malleret-Joinville, 94114 Arcueil Cedex, France \\ 2 Université de Marne la Vallée, LaM EA2545, 5 boulevard Descartes, Champs sur Marne, 77454 Marne la Vallée Cedex, France
}

Reçu le 21 novembre 2006, accepté le 9 août 2007

\begin{abstract}
Résumé - L'étude présentée dans cet article concerne le problème industriel de l'usure dans les contacts roulants sous charge. La déformation locale de la zone de contact associée à l'existence d'efforts tangentiels conduit à du micro glissement et de l'usure superficielle qui «creuse » la piste de roulement. D'un point de vue industriel, cette usure détériore le fonctionnement des machines pilotées par des systèmes « camegalet ». Dans le prolongement de l'étude présentée dans [1] les auteurs proposent une démarche qui permet de suivre l'évolution de l'usure au cours du temps tout en prenant en compte les dispersions expérimentales observées sur banc d'essai. Les méthodes présentées ici, peuvent être appliquées au dimensionnement des roulements et engrenages par exemple, et plus généralement à tout mécanisme dans lequel il y a du roulement faiblement lubrifié entre deux solides. Dans le cadre de cet article on se limite au cas stationnaire pour lequel la charge normale et la vitesse de renouvellement du contact n'évoluent pas au cours du temps. C'est dans ce cadre que nos essais d'usure ont été réalisés et l'objectif de cette étude est de simuler ces essais. La démarche suivie prend en compte les dispersions de positionnement des pièces, les dispersions mesurées sur l'effort de contact et les incertitudes du coefficient de frottement au travers d'une approche probabiliste.
\end{abstract}

Mots clés : Contact roulant / semi-hertzien / usure / simulation numérique / approche stochastique

Abstract - Probabilistic simulation of wear in loaded rolling contact. The study presented in this paper deals with the wear phenomena which occurs during loaded rolling contact in industrial machinery. The local deformation of the local contact zone associated to the existence of tangential load generates micro slips and superficial wear. This damage leads to machinery diseases, for example in cam-roller system the kinetic of the machine may not be as smooth as initially needed. As a complement of the previous presentation in [1] authors show simulation of the wear profile which is due to successive passages of the roller on the cam path. This simulation is managed taking into account the dispersion of not well known parameters by a stochastic approach. These methods may be applied to other mechanical applications such as roller bearing analysis, for example. In this paper, we restrict our study to the quasi-stationary of a roller on a cylindrical specimen case where normal load, geometry and speed are constant during simulation. The study takes into account experimental dispersions via the construction of probabilistic density function associated with uncertain parameters such as the relative position of solids or contact characteristics such as the coefficient of friction $\mu$.

Key words: Rolling contact / semi-Hertzian method / wear / numerical simulation / stochastic approach

\section{Introduction}

Les machines de production utilisant des systèmes came-galet nécessitent de bien contrôler l'usure afin d'éviter certains dysfonctionnements. Pour cela, disposer d'outils d'analyse permettant de mettre en évidence

\footnotetext{
a Auteur pour correspondance :

luc.chevalier@univ-mlv.fr
}

l'influence de certains paramètres sur la tenue à l'usure des cames apporte une aide précieuse à la conception, surtout si l'on considère l'augmentation des sollicitations cinématiques et mécaniques dues à l'accroissement des cadences de production. Pour réaliser des comparaisons sur la tenue à l'usure de différents matériaux en fonction de différents paramètres, un banc d'essai a été développé 
pour recréer les conditions de contact roulant entre les galets et les cames.

Nous proposons dans cet article une démarche pour simuler ces essais. Pour cela, après un bref rappel d'une première étude, nous présentons des résultats expérimentaux d'usure. La dispersion de chaque paramètre important $\mathrm{y}$ est quantifiée par des mesures et une analyse statistique. Dans la partie suivante nous proposons un modèle local d'usure, dérivé des travaux d'Archard, ainsi qu'une démarche de simulation de l'usure pour prendre en compte l'évolution des profils usés en fonction du nombre de cycles. Nous présentons également dans cette partie les méthodes simplifiées et rapides utilisées pour résoudre le problème du contact roulant entre deux solides élastiques. Nous présentons finalement dans la dernière partie, l'approche probabiliste retenue pour prendre en compte les dispersions expérimentales dans la simulation de l'usure. Cette approche nous permet de déduire la fiabilité associée aux résultats de simulation.

\section{Usure dans les contacts roulants}

\subsection{Détérioration des pistes de roulement par usure superficielle}

Des mesures d'usure à l'aide d'un comparateur et des mesures de dureté post mortem ont permis de réaliser des comparatifs de tenue à l'usure et de tenue à la fatigue de différents aciers pour la fabrication des cames de souffleuses de bouteilles plastiques [2].

Sur le banc d'essai, la came est assimilée à une éprouvette cylindrique (aussi nommée « rondin ») de diamètre $\phi_{r}=50 \mathrm{~mm}$. Les galets sont les mêmes que ceux utilisés sur les souffleuses de bouteilles plastiques : galet de roulement INA PWTR 17.2RS. Ce dispositif expérimental permet de faire varier la vitesse de rotation du rondin, l'effort de contact $F$ et le positionnement angulaire du galet sur l'éprouvette cylindrique. Une vue 3D simplifiée du montage est présentée en figure 1.

La charge imposée est mesurée par un capteur de compression SCAIME K1613 d'une capacité maximale de 5000 N. L'électronique de mesure utilisée est un conditionneur afficheur pour capteurs à pont de jauges : conditionneur/afficheur SCAIME série IMS. Nous notons $\varphi_{x}$ et $\varphi_{z}$ les défauts angulaires de parallélisme entre l'axe du galet et l'axe du rondin tels que définis sur la figure 2 .

Les essais réalisés, dans les conditions de vitesse et pression de contact analogues à celles des contacts dans les souffleuses, sur des éprouvettes de 42CrMo4 ayant subi un traitement thermique de trempe par induction sur une profondeur de l'ordre de $5 \mathrm{~mm}$ montrent des relevés de microdureté qui ne présentent pas de variation significative par rapport à la dureté initiale. Par contre, sur des photographies de la surface de la piste de roulement on voit clairement des variations géométriques dues à l'usure superficielle. La figure 3 illustre ces résultats.

Nous remarquons que la dureté reste constante ( $H_{v}=700 \mathrm{MPa}$ en moyenne) jusqu'à la profondeur de $4 \mathrm{~mm}$ environ (épaisseur du traitement thermique).

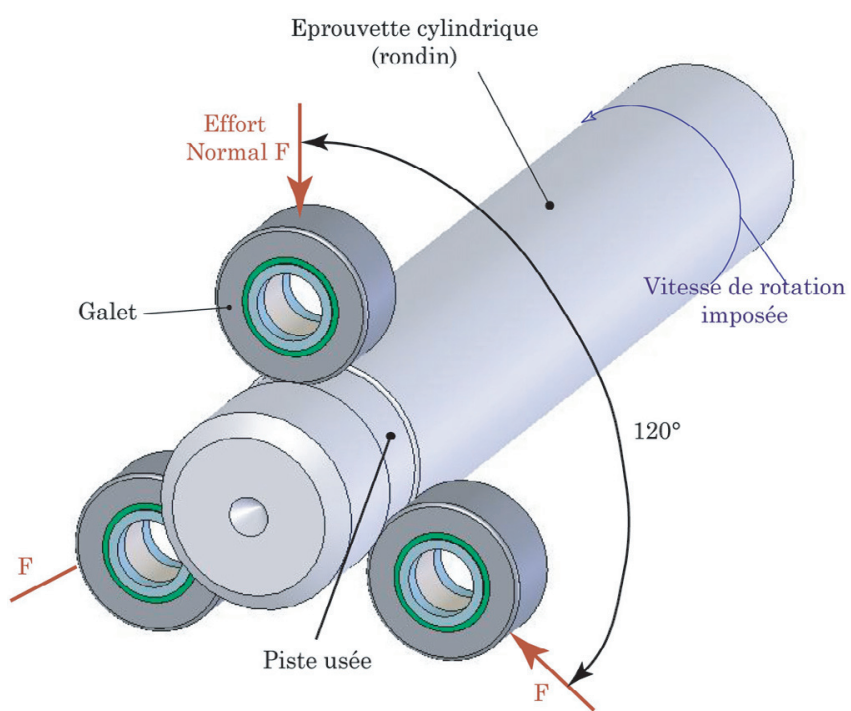

Fig. 1. Vue 3D simplifiée de principe du positionnement des galets par rapport à l'éprouvette cylindrique.

Cela correspond à la zone traitée thermiquement. Le mode de détérioration observé sur cette piste de roulement est de l'usure abrasive. La présence de rayures suivant la direction de roulement (petits sillons parallèles) confirme le type d'usure du matériau traité. Nous observons également la présence de « marches » sur les bords de la piste sans la présence de bourrelets; ce qui montre bien l'enlèvement de matière suite aux passages répétés des galets et l'absence de plasticité. Ces résultats ont été présentés dans [2] et c'est l'analyse des dispersions lors des essais et la simulation de ce mode de détérioration que nous détaillons dans la suite.

\subsection{Banc d'essai d'usure : importantes dispersions expérimentales}

La mesure de l'usure par «profilométrie » permet de suivre l'évolution du profil usé en fonction du nombre de cycles. Les profils mesurés sont très rugueux et pour améliorer la lisibilité, l'évolution des sections d'usure est construite à partir d'une moyenne mobile sur cinq points de chaque profil. L'usure maximale $\left(U_{\text {maxi }}\right)$ est la profondeur maximale du profil usé moyenné comme le montre la figure 4.

Nous présentons les résultats obtenus pour trois essais. Ils ont été réalisés dans des conditions réputées identiques : charge de $300 \mathrm{~N}$, vitesse de rotation du rondin de 1000 tr.min ${ }^{-1}$ (soit une vitesse de renouvellement du contact de $V=2,6 \mathrm{~m} . \mathrm{s}^{-1}$ ), angles de dérive et de spin nuls. L'évolution de l'usure maximale de ces trois essais est résumée sur la figure 5.

Le premier constat est que les résultats sont assez dispersés. Les mesures d'usure entre les essais 2 et 3 sont bien groupées entre $10^{6}$ et $1,5 \times 10^{6}$ cycles. À certains instants d'essai, les écarts entre l'usure maxi d'un essai à l'autre peuvent aller du simple au triple. Nous constatons 


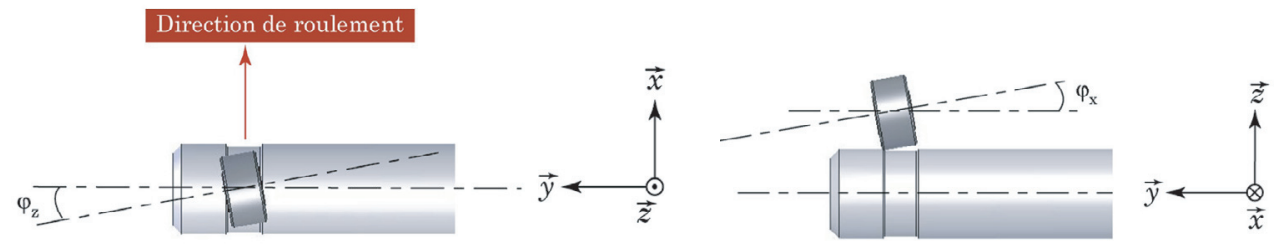

Fig. 2. Mise en évidence de l'angle de dérive (également appelé angle de lacet) et de l'angle de spin (également appelé angle de pivotement)
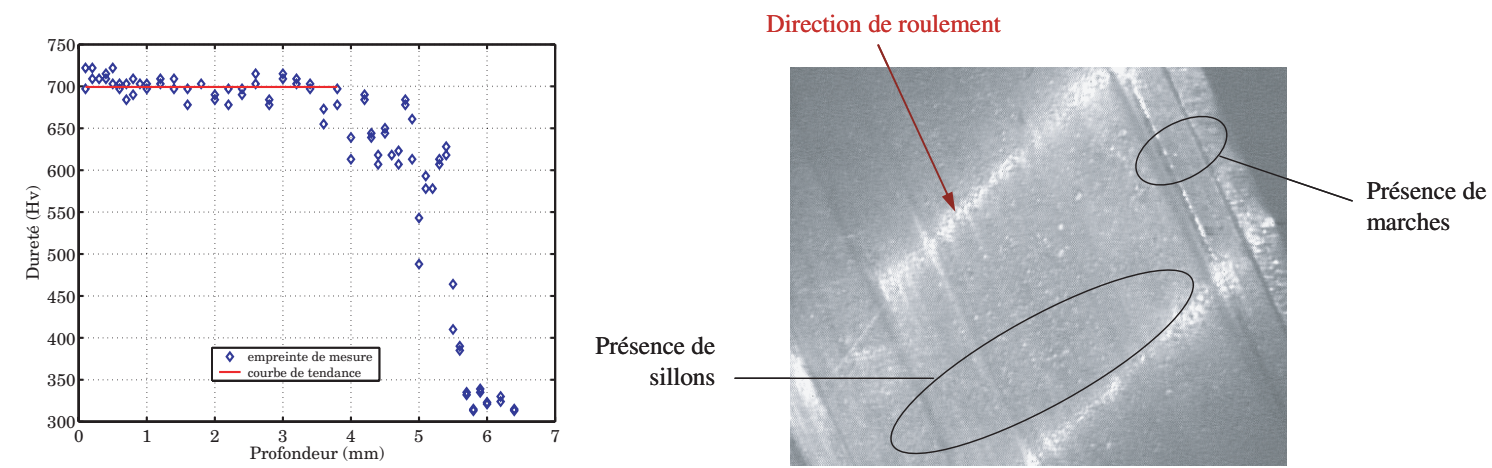

Fig. 3. Évolution de la dureté en fonction de la profondeur et vue de la piste de roulement.

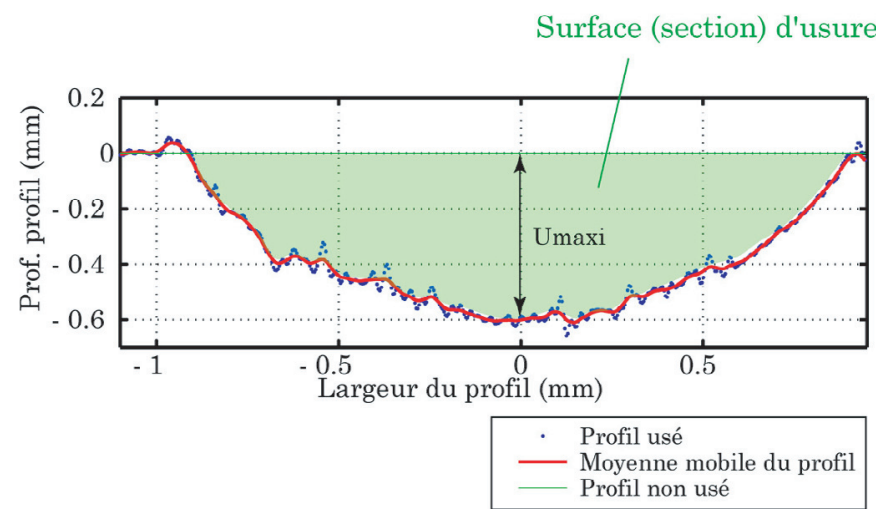

Fig. 4. Définition de l'usure maximale $U_{\operatorname{maxi}}$.

sur la figure 6 que pour les trois essais, les profils mesurés sont dissymétriques. Cette dissymétrie indique que le galet n'est pas parfaitement positionné angulairement sur le rondin. Notamment, l'angle de spin n'est donc pas nul.

La dispersion entre les essais est particulièrement importante au début de l'essai. La différence entre les profils est assez faible ensuite entre les essais 2 et 3 avec des largeurs de profils similaires et des variations de 20 à $30 \%$ sur les profondeurs d'usure. Par contre, les résultats de l'essai 1 sont toujours en deçà des essais 2 et 3 en terme de largeur de profil et de profondeur d'usure.

L'origine de ces dispersions est multiple : le chargement normal, le positionnement du galet sur le rondin, la traînée de chaque galet, le coefficient de frottement, la coexistence de trois galets roulant dans la même trace et pouvant être réglés différemment.

L'évolution du chargement de chaque boîtier (au nombre de trois) au cours du temps a été relevée pour

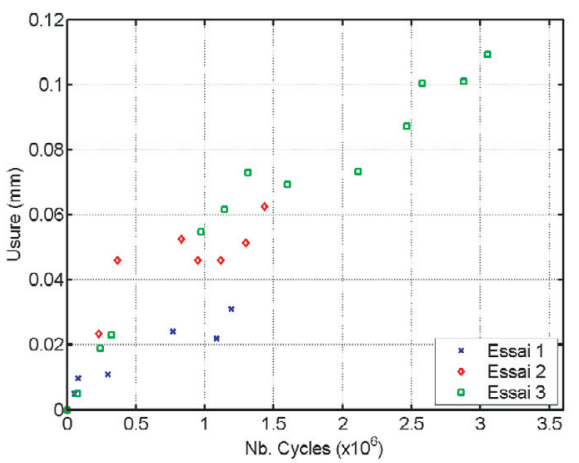

Fig. 5. Évolution de l'usure maximale en fonction du nombre de cycles pour les trois essais.

tous les essais réalisés. Pour chaque essai, une augmentation suivie d'une diminution ou non de l'effort est observée. La dissipation énergétique au niveau de la surface de contact entraîne une augmentation de la température. Il s'en suit un échauffement du boîtier et de l'ensemble du montage au voisinage de la surface de contact. Cet échauffement s'accompagne d'une dilatation des corps et induit un chargement supplémentaire du fait de la raideur élevée de l'empilement de rondelles élastiques. C'est une explication possible des « remontées » de charge après chaque arrêt de l'essai.

Chaque effort est tracé sur le même graphique (Fig. 7) pour mettre en évidence la diminution de l'effort avec l'usure. Compte tenu de la raideur du montage de rondelles Belleville, cette diminution est directement proportionnelle à l'usure. En effet une usure de $0,1 \mathrm{~mm}$, par exemple, entraîne une diminution de l'effort de 22 \% La raideur étant connue, l'évolution de l'effort pourra s'écrire 

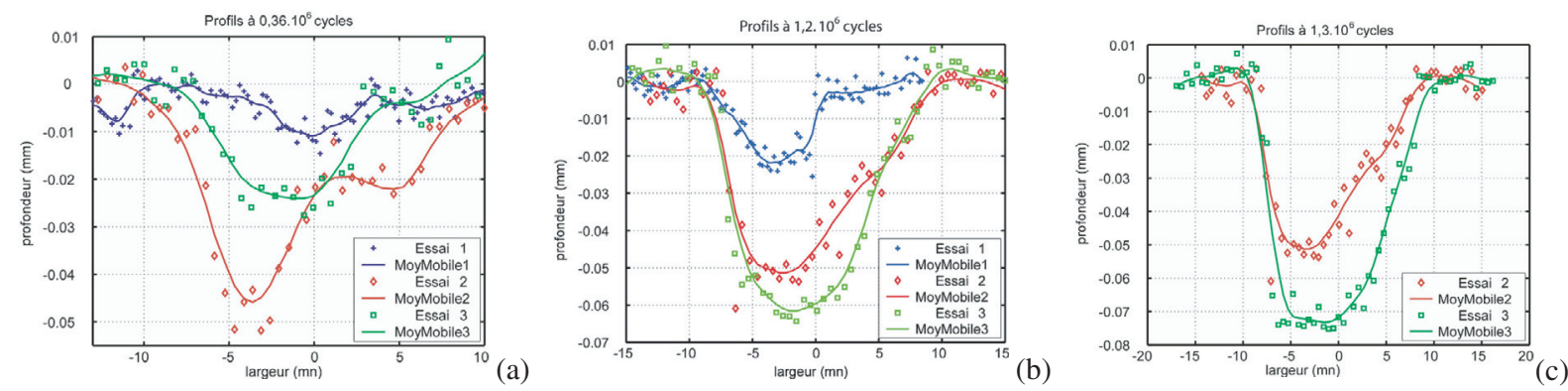

Fig. 6. Comparaisons des profils usés pour différents instants.
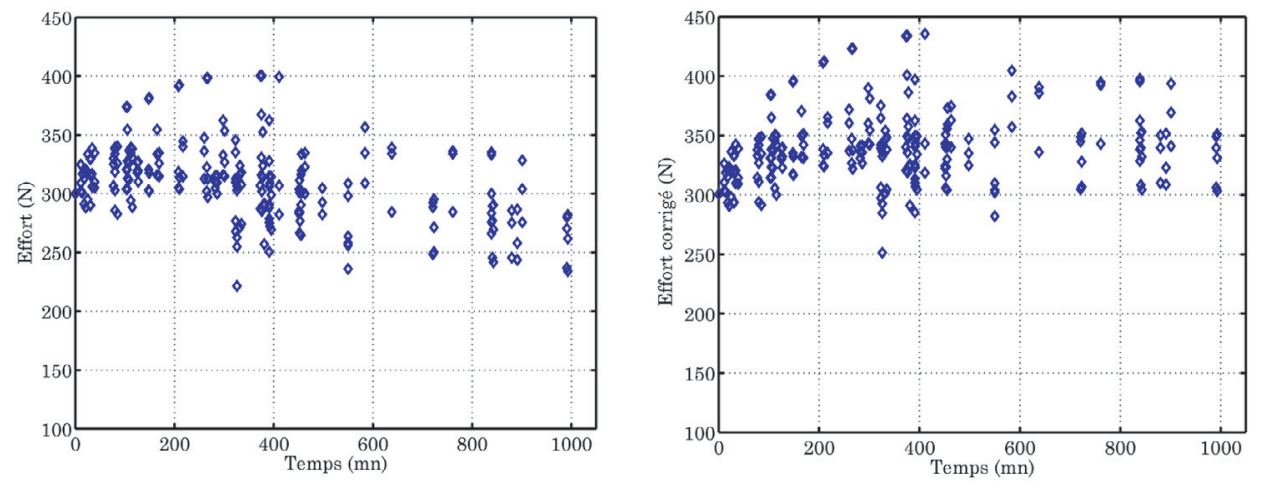

Fig. 7. Évolution des efforts de chaque boitier en fonction du temps d'essai pour six essais réputés identiques (à gauche) et «redressé » pour prendre en compte le déchargement des rondelles élastiques due à l'usure (à droite).

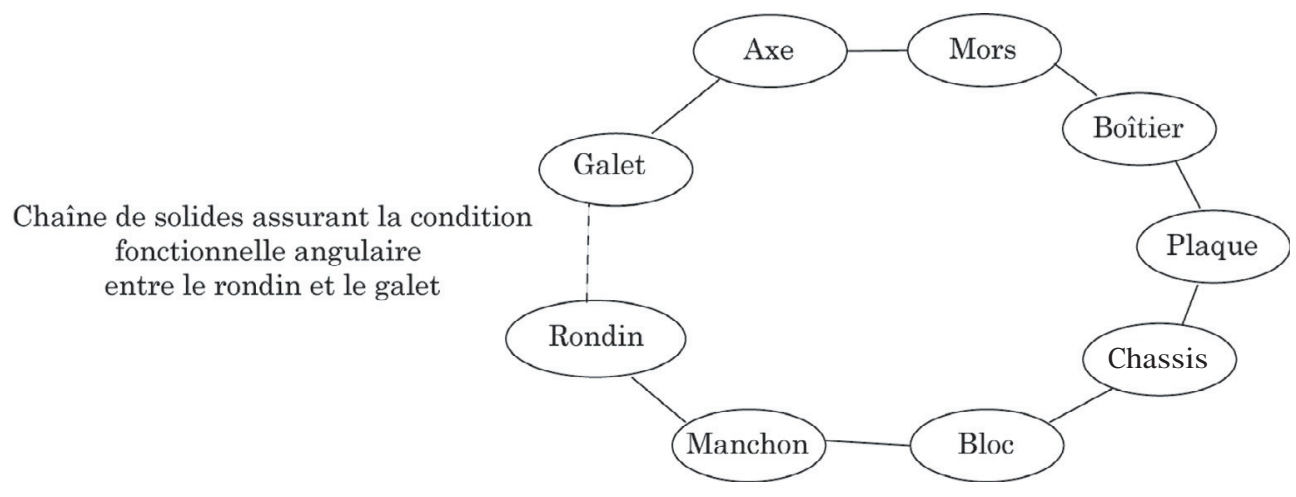

Fig. 8. Graphe de liaisons assurant le positionnement angulaire du galet sur le rondin.

par :

$$
F(t)=F_{0}-k_{\mathrm{m}} u_{\max }(t) \text { avec } k_{m}=670 \mathrm{~N} \cdot \mathrm{mm}^{-1}
$$

où $k_{\mathrm{m}}$ est la raideur de l'assemblage de rondelles élastiques, $F_{0}$ est la charge initiale (300 $\mathrm{N}$ dans notre cas) et $u_{\max }(t)$ est la profondeur usée maximale du rondin à l'instant $t$.

Pour quantifier la dispersion existant sur cet effort lors des essais on découple l'effet de l'usure en « redressant » les valeurs d'effort. Nous modélisons l'évolution de $u_{\max }(t)$ par une fonction parabolique dont les coefficients sont identifiés sur les tracés superposés de 3 essais présentés plus haut. Une fois redressé, nous pouvons calculer la valeur moyenne de la charge normale $m_{\mathrm{F}}=340 \mathrm{~N}$ et un écart type $\sigma_{\mathrm{F}}=33 \mathrm{~N}$ due aux variations de température et autres effets mal maîtrisés.
Le guidage des galets est assuré par une chaîne de pièces mécaniques assemblées les unes aux autres. Les ajustements des différents assemblages de la chaîne sont tels qu'ils rendent incertain le positionnement angulaire du galet par rapport au rondin.

L'incertitude du positionnement angulaire du galet (Fig. 8) par rapport au rondin dépend de la chaîne de solides qui vont intervenir sur ce positionnement. Le cumul des défauts angulaires de chaque pièce de la branche permet de quantifier un défaut global de mise en position du galet par rapport au rondin. Compte tenu du mode de construction du banc d'essai : ensemble mécano-soudé; des états de surface et des défauts de positionnement, nous pouvons estimer la tolérance sur l'angle de dérive $\varphi_{z} . \grave{A}$ partir d'ordre de grandeur de positionnement en 


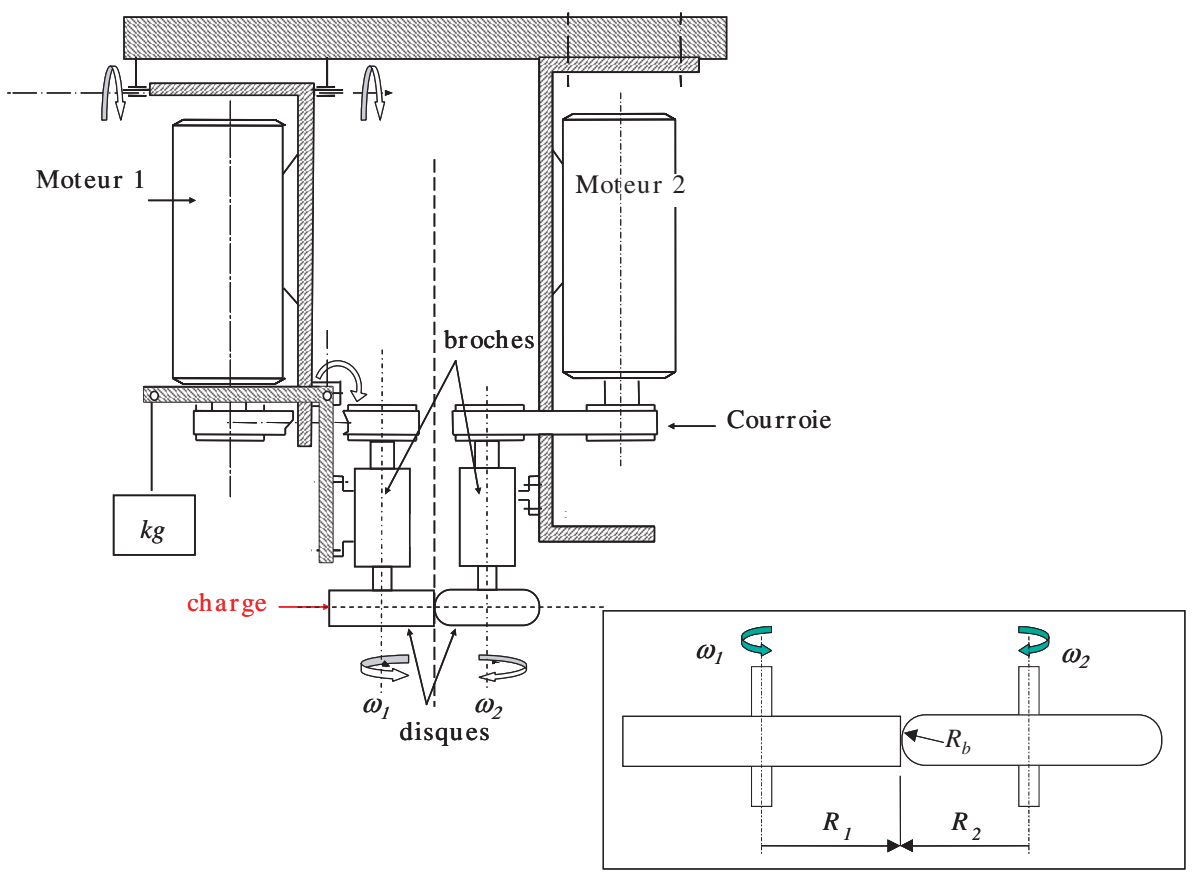

Fig. 9. Schéma du tribomètre bidisque du LISMMA. Les vitesses de rotation de chaque moteur sont contrôlées.

fonction du type de construction, l'angle de dérive vaut : $\varphi_{z}=0 \pm 0,02 \mathrm{rad}$.

Nous avons constaté sur la figure 6 que les profils d'usure sont dissymétriques. Cette dissymétrie est due à l'angle de pivotement $\varphi_{x}$ qui est non nul. Un moyen simple de quantifier cet angle est de chercher la position angulaire du galet qui minimise un écart entre le profil du galet et les profils usés. Nous avons appliqué cette méthode aux profils d'usure disponibles; la valeur moyenne de l'angle de pivotement est estimée à $3,2 \times 10^{-3}$ rad avec un écart type de $1,4 \times 10^{-3} \mathrm{rad}$.

\subsection{Mesure du coefficient de frottement par des essais bi-disques}

Outre les caractéristiques géométriques et cinématiques du banc et des propriétés élastiques des matériaux en contact, il est nécessaire de connaître quelques propriétés du contact lui-même (coefficient de frottement $\mu$ et facteur d'usure $K$ ) pour mener à bien la simulation. L'utilisation d'un tribomètre bi-disques permet de réaliser des mesures de coefficient de frottement en présence de glissement. Les essais ont été effectués au Laboratoire d'Ingénierie des Systèmes Mécaniques et des Matériaux (LISMMA) de SupMéca à Saint Ouen. Le tribomètre bi-disques utilisé a été développé pour l'étude du comportement des roulements de turbopompes en ambiance cryotechnique [3].

Le tribomètre se compose de deux broches mécaniques entraînées en rotation par des courroies. Sur chaque broche est monté un disque (ces disques peuvent être cylindriques ou sphériques comme sur le schéma de la
Fig. 9). Deux moteurs sans balai à aimants permanents de forte puissance massique sont destinés à entraîner à vitesse variable les deux broches (vitesse de 1800 à 11000 tr.min ${ }^{-1}$ ), d'un dispositif de chargement par masses capables d'appliquer un effort normal jusqu'à $710 \mathrm{~N}$ entre les deux disques, de suspensions articulées réparties, d'une part au niveau d'une des broches et de son moteur pour pouvoir mesurer l'effort tangentiel généré au contact des disques, et d'autre part au niveau de cette même broche afin de s'affranchir au maximum des faux ronds des disques, des moyens de mesure des vitesses de rotation de chacune des broches et de l'effort tangentiel lié au frottement.

Les différentes données sont enregistrées en continu pendant toute la durée de l'essai. Les calculs du coefficient de frottement (rapport de la force tangentielle mesurée sur la force normale imposée) et du taux de glissement sont effectués tout au long de l'essai. Le taux de glissement est défini par la relation :

$T_{\mathrm{g}}=\frac{\Delta V}{V_{\text {moy }}}$ avec $\Delta V=V_{2}-V_{1}$ où $\left\{\begin{array}{l}V_{1}=R_{1} \omega_{1} \\ V_{2}=R_{2} \omega_{2} \\ V_{\text {moy }}=\left(V_{2}+V_{1}\right) / 2\end{array}\right.$

Le disque 1 est un disque cylindrique (géométrie du rondin) en $42 \mathrm{CrMo} 4$ ayant subi une trempe superficielle sur $0,4 \mathrm{~mm}$. Le rayon $R_{1}$ vaut $25 \mathrm{~mm}$. Le disque 2 (disque bombé) possède une géométrie se rapprochant de la géométrie du galet en 100Cr6 : rayon $R_{2}$ de $35 \mathrm{~mm}$ et un bombé $R_{\mathrm{b}}$ de $300 \mathrm{~mm}$. Dans le cas du banc d'essai à galets, la pression de contact est de l'ordre de $600 \mathrm{MPa}$. Pour obtenir une pression de contact du même ordre avec les géométries des disques, l'effort à appliquer doit être de $185 \mathrm{~N}$ environ. 

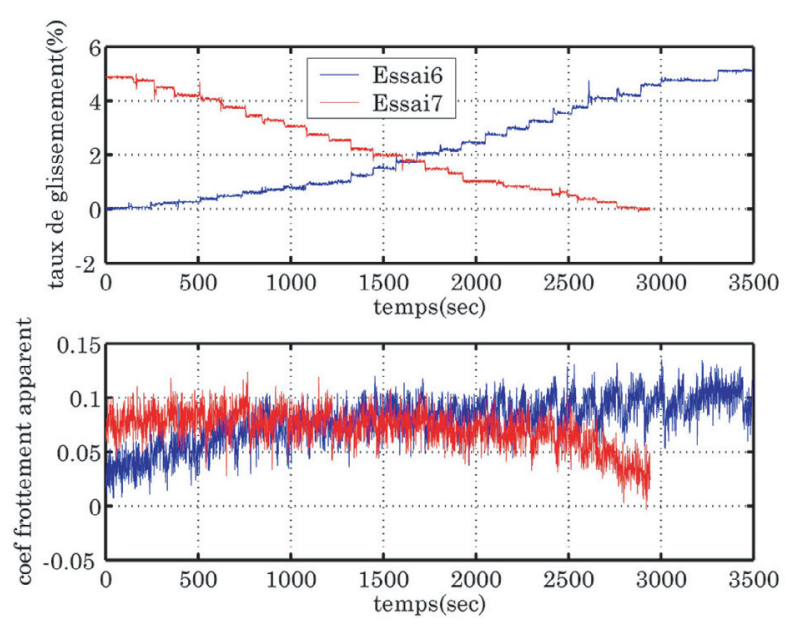

Fig. 10. Mesure du coefficient de frottement dans le cas d'essai bidisque lubrifié par de la graisse au savon complexe de lithium avec additif EP à base d'huile minérale.

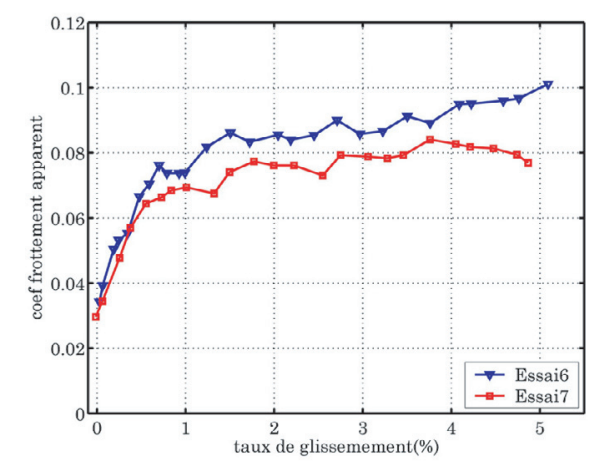

Fig. 11. Mesure du coefficient de frottement en régime lubrifié en fonction du taux de glissement.

La vitesse de rotation de la broche 2 est maintenue à 2700 tr. min $^{-1}$. La vitesse de rotation de la broche 1 est imposée à 1931 tr.min ${ }^{-1}$ (cas du roulement sans glissement) pour décroître jusqu'à 1800 tr.min ${ }^{-1}$. Avant de commencer chaque essai, les broches, les disques et l'environnement proche du contact sont nettoyés au trichloréthylène pour éliminer tout polluant susceptible de se glisser dans le contact. Des essais bi-disques lubrifiés par une graisse identique à celle du galet (graisse au savon complexe de lithium avec additif EP à base d'huile minérale) sont présentés sur les figures 10 et 11 . La fréquence de lubrification est toujours la même. À chaque changement de palier, une pression exercée sur la seringue injecte un volume de graisse et lubrifie la piste de roulement. La graisse est en partie éjectée, mais une partie adhère aux disques.

Deux essais ont été effectués en imposant des taux de glissement croissants dans un premier temps et décroissants dans un second temps. Dans le cas de l'essai $6, T_{\mathrm{g}}$ croît de 0 à $5 \%$ alors que dans le cas de l'essai $7, T_{\mathrm{g}}$ décroît de $5 \%$ à 0 comme le montre la figure 10 .

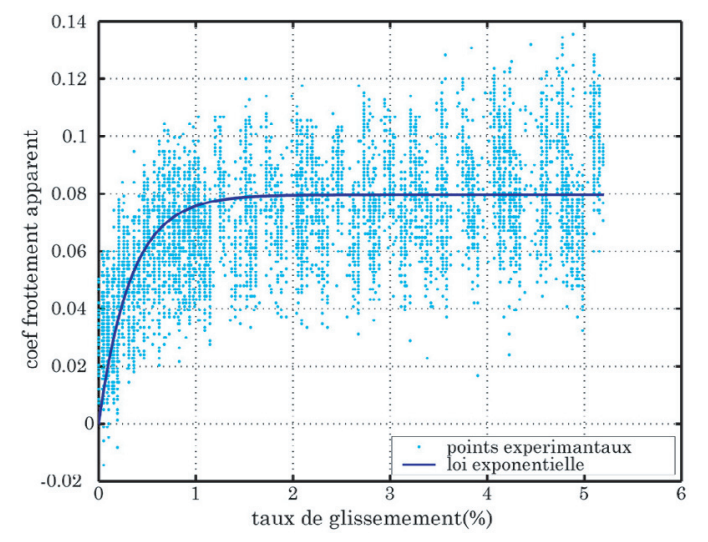

Fig. 12. Représentation de la loi exponentielle identifiée sur les mesures de coefficient de frottement réalisées en présence de lubrifiant.

La figure 11 montre que le coefficient de frottement croît jusqu'à atteindre une valeur stable. La dispersion sur les mesures est importante comme le montre la valeur de l'écart type $\left(\sigma_{\mu}=0,02\right)$ des mesures devant la valeur moyenne $\left(m_{\mu}=0,08\right)$. À partir des mesures de coefficient de frottement et en supposant que l'évolution du coefficient de frottement est asymptotique, nous identifions une loi exponentielle, analogue à celle utilisée dans $[4,5]$, présentée par la relation :

$$
\mu_{a \operatorname{lub}}\left(T_{\mathrm{g}}\right)=\mu_{a \operatorname{lub}}^{\infty}\left(1-\exp \left(-\lambda_{\mathrm{lub}} T_{\mathrm{g}}\right)\right)
$$

où $\mu_{a \text { lub }}$ est le coefficient de frottement apparent, $\mu_{a \text { lub }}^{\infty}$ est la valeur asymptotique du coefficient de frottement et $\lambda_{\text {lub }}$ est une constante à identifier. Ces deux dernières valeurs valent dans le cas d'un frottement lubrifié : $\mu_{a l u b}^{\infty}=0,0796$ et $\lambda_{\text {lub }}=3,03$.

Cette loi exponentielle est tracée sur la figure 12 .

Nous pouvons noter que la dispersion autour de la loi exponentielle est importante. Le coefficient de frottement peut varier de 0,02 à 0,14 pour des contacts lubrifiés acier sur acier. La lubrification entre le galet et le rondin est un paramètre difficile à maîtriser sur le banc à galets. En effet, les essais sont réputés « à sec» mais la graisse contenue dans le galet qui s'échauffe au cours de l'essai, se fluidifie et coule sur la piste de roulement. Ceci génère une lubrification non souhaitée qui perturbe fortement les conditions expérimentales. Ainsi, un essai commencé « à sec » est vite réalisé en régime lubrifié sans qu'il soit possible de quantifier cette perte de graisse dans le galet. Ainsi, le volume de lubrifiant répandu sur la piste de roulement ne peut pas être estimé.

Les coulées de lubrifiant dans la zone de contact ne permettent pas de définir précisément les conditions de frottement. La valeur du coefficient de frottement est donc incertaine et les essais bi-disques lubrifiés nous ont permis de quantifier cette incertitude par une valeur moyenne et un écart type sur le coefficient de frottement. 


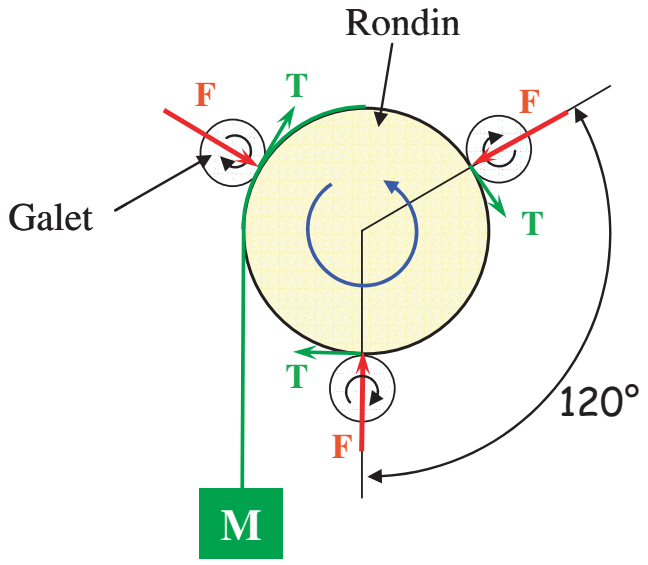

Fig. 13. Présentation du dispositif permettant la mesure du couple résistant en fonction de l'effort normal.

\subsection{Estimation du taux de glissement $T_{\mathrm{g}}$}

La détermination du taux de glissement $T_{\mathrm{g}}$ (ou pseudoglissement longitudinal $v_{x}$ ) est importante pour les simulations des essais d'usure du banc à galets. Dans ce cas, seule la vitesse du rondin est contrôlée. Il n'y a pas exactement roulement sans glissement entre le galet et le rondin. Le taux de glissement entre le galet et le rondin dépend du couple résistant au niveau du galet. Ce couple résistant possède deux origines : le frottement interne dans les galets entre le joint à lèvre et la bague intérieure, la traînée due à la graisse environnant les rouleaux et s'opposant à leurs déplacements.

L'encombrement du banc d'essai ne permet pas l'installation d'un tachymètre pour mesurer la vitesse de rotation du galet. Par contre, nous pouvons mesurer le couple résistant du galet sous charge par un montage très simple. Le principe de la mesure est présenté figure 13.

Un système de masse permet de mesurer le couple résistant en fonction de l'effort normal $F$ appliqué sur le rondin. Le principe est d'augmenter la masse $M$, jusqu'à créer un couple $C_{\mathrm{M}}$ plus important que le couple résistant $C_{\mathrm{r}}$ du galet, et entraîner la descente de la masse par gravité. L'effort tangent $T$ s'opposant au roulement du galet et la masse $M$ est donc déterminé par l'expression suivante :

$$
T=\frac{g M}{3}
$$

$g$ est l'accélération de la pesanteur. Onze mesures ont été réalisées avec des galets neufs. L'effort tangentiel moyen $T$ vaut $3,2 \mathrm{~N}$ pour un écart type de $0,22 \mathrm{~N}$ et l'effort normal $F$ vaut $300 \mathrm{~N}$. Le rapport $\mu_{r}=T / F=0,011$. La dispersion est pratiquement nulle : l'écart type par rapport à la valeur moyenne est de 6,5\%. Cependant, au cours des essais d'usure sur le banc à galets, les changements des conditions de frottement auront une incidence sur le taux de glissement et nous allons utiliser les mesures de coefficient de frottement réalisées lors des essais bi-disques pour estimer le taux de glissement. Nous considérons donc $v_{x}$ (ou $T_{\mathrm{g}}$ ) comme une variable dispersée. La construction de cette variable est détaillée sur la figure 14 et la procédure est explicitée par la suite.

(1) À partir des domaines de variation, des valeurs moyennes et des écarts type de $\mu_{\mathrm{a}}$ et $\mu_{\mathrm{r}}$, nous construisons les densités de probabilité associées à chaque variable. Les domaines de variation de chaque variable sont choisis pour englober la plus grande dispersion. Ils sont compris entre la valeur moyenne et $\pm \pi$ fois l'écart type.

(2) Un tirage $i »$ de Monte Carlo est effectué pour déterminer une valeur de $\mu_{\mathrm{a}}: \mu_{\mathrm{a}}^{i}$.

(3) Nous considérons qu'il y a couplage entre la valeur de $\mu_{\mathrm{a}}$ et la pente $\lambda$ à l'origine. Pour chaque valeur tirée $\mu_{\mathrm{a}}^{i}$, il faut définir le $\lambda_{i}$ correspondant. En notation indicielle, le modèle de frottement exponentiel s'écrit : $\mu_{\mathrm{a}}\left(T_{\mathrm{g}}\right)=$ $\mu_{\mathrm{a}}^{i}\left(1-\exp \left(-\lambda_{i} T_{\mathrm{g}}\right)\right)$.

(4) À partir de la densité de probabilité sur le coefficient $\mu_{\mathrm{r}}$, un tirage de Monte Carlo $\mu_{\mathrm{r}}^{i}$ est réalisé.

(5) La valeur de $T_{\mathrm{g}}^{i}$, correspondant à un tirage « $i »$, est calculée par la relation :

$$
T_{\mathrm{g}}^{i}=\frac{1}{\lambda_{i}} \ln \left(1-\frac{\mu_{\mathrm{r}}^{i}}{\mu_{\mathrm{a}}^{i}}\right)
$$

Après une étude de convergence de la méthode et 20000 tirages, nous estimons les valeurs statistiques du taux de glissement à :

$$
\begin{aligned}
m_{T_{\mathrm{g}}} & =6 \times 10^{-2} \% \\
\sigma_{T_{\mathrm{g}}} & =4,6 \times 10^{-2} \%
\end{aligned}
$$

La dispersion est très importante par rapport à la valeur moyenne.

\section{Simulation de l'évolution de l'usure superficielle}

\subsection{Modélisation de l'usure superficielle observée lors des essais}

L'usure est considérée comme un phénomène dissipatif associé à des détachements de particules : il s'agit d'une perte de matière. Les corps que l'on met originellement en contact vont agir, lors de mouvements particuliers, en tant que source de troisième corps : on parle alors de tribosystème. Le troisième corps n'est pas nécessairement composé de particules; il peut être fluide, solide, métallique, organique ou minéral. Dans notre cas, le troisième corps sera majoritairement composé de graisse fluide d'une part et de particules solides d'autre part. La graisse provient des galets dont l'étanchéité n'est pas parfaite, et les particules solides proviennent de l'arrachement de matière des deux autres corps en contact. Ces deux quantités vont varier au cours du temps. La quantité de graisse qui coule sur la piste va dépendre du volume de graisse emprisonné dans le galet. Aux particules déjà présentes dans le troisième corps et pas encore évacuées, vont s'ajouter de nouvelles particules arrachées et se soustraire des particules qui seront éjectées du contact. Des auteurs [6-8] 


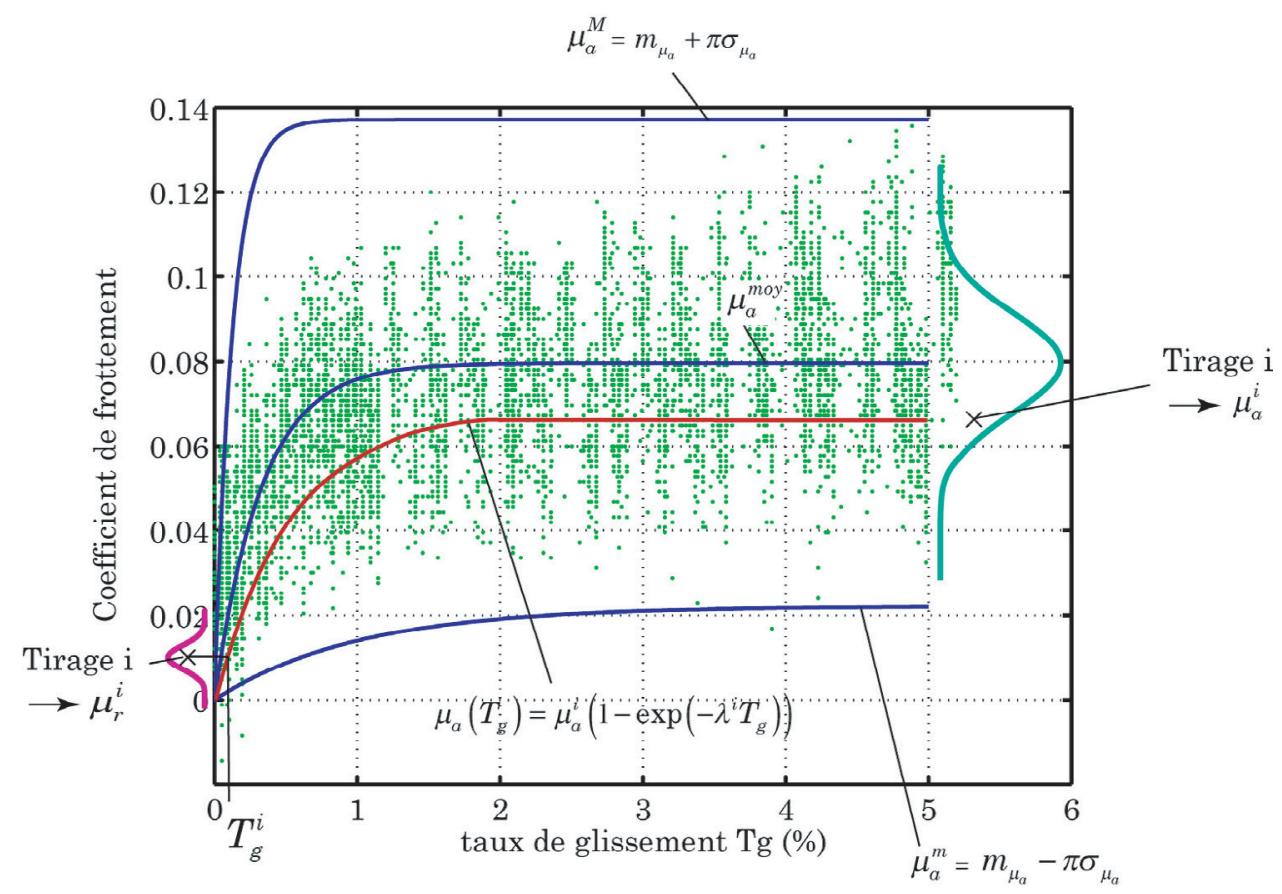

Fig. 14. Construction de la dispersion autour de la valeur moyenne du taux de glissement. Construction de la dispersion du taux de glissement.

utilisent des modèles basés sur la méthode des éléments discrets (DEM) couplés à des critères d'arrachement de particules pour modéliser l'usure et le comportement de ce troisième corps. Cette approche permet de bien décrire les phénomènes physiques à l'origine des flux entrants et sortants de l'interface entre les deux corps en contact. Cependant les méthodes mises en place sont gourmandes en temps de calcul et ne sont pas adaptées à la simulation rapide de l'évolution de l'usure pour un grand nombre de cycles. C'est pourquoi, dans notre étude, la modélisation du troisième corps n'est volontairement pas prise en compte et les propriétés de l'interface sont définies par le coefficient de frottement mesuré par les essais bi-disques en régime lubrifié.

Pour modéliser l'usure, de nombreux auteurs de ces dernières décennies se sont appuyés sur l'étude réalisée par Archard [9]. Ce fut le premier à formaliser mathématiquement l'évolution de l'usure. Cette loi relie le volume d'usure $W$ à la charge normale appliquée $F$, à la longueur de glissement $l$ et à l'inverse de la dureté $H$ du matériau le plus tendre. Elle s'écrit :

$$
W=K \frac{F l}{H}
$$

$K$ est le coefficient d'usure; c'est un nombre sans dimension dépendant du couple de matériaux en contact, de la lubrification, de la vitesse de glissement et de la pression de contact comme montré par [10]. La figure 15 illustre le principe de ce modèle d'usure.

Il est à noter que $K$ est souvent utilisé pour séparer l'usure douce de l'usure sévère et qu'il découle d'une série d'expérimentations. Des études plus récentes [10-18], reprennent cette loi sous diverses formes : globales ou locales

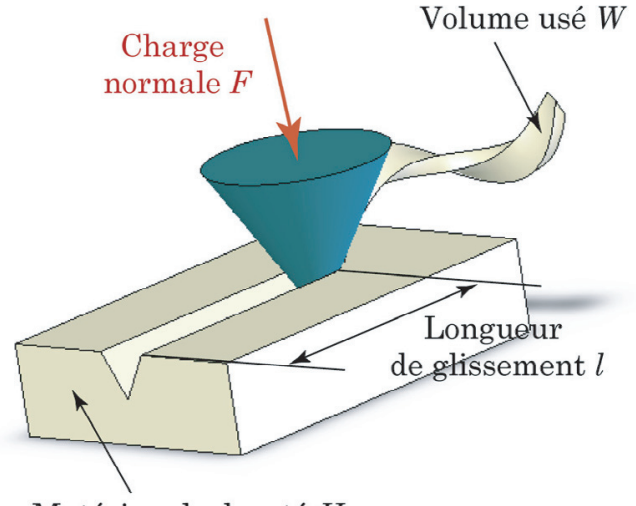

Matériau de dureté $H$

Fig. 15. Illustration du modèle d'Archard.

suivant le cas. Elles ne prennent généralement pas en compte les répartitions de la vitesse de glissement et des forces surfaciques tangentielles sur la surface de contact. Nous proposons une nouvelle écriture locale du modèle d'usure issu du modèle d'Archard qui, sous sa forme instantanée, fait apparaître la vitesse de glissement et qui, dans la zone de glissement fait intervenir les forces surfaciques tangentielles.

$$
\frac{\mathrm{d} W}{\mathrm{~d} t}=\dot{W}=\frac{K}{H} F V_{\mathrm{g}} \operatorname{car} \frac{\mathrm{d} l}{\mathrm{~d} t}=i=V_{\mathrm{g}}
$$

$\dot{W}\left[\mathrm{~m}^{3} \cdot \mathrm{s}^{-1}\right]$ est le taux d'usure c'est-à-dire le volume usé par unité de temps. À l'échelle du contact, la vitesse glissement $V_{\mathrm{g}}$ n'est pas globale mais locale : on la note $w_{\mathrm{g}}(x, y)$. 

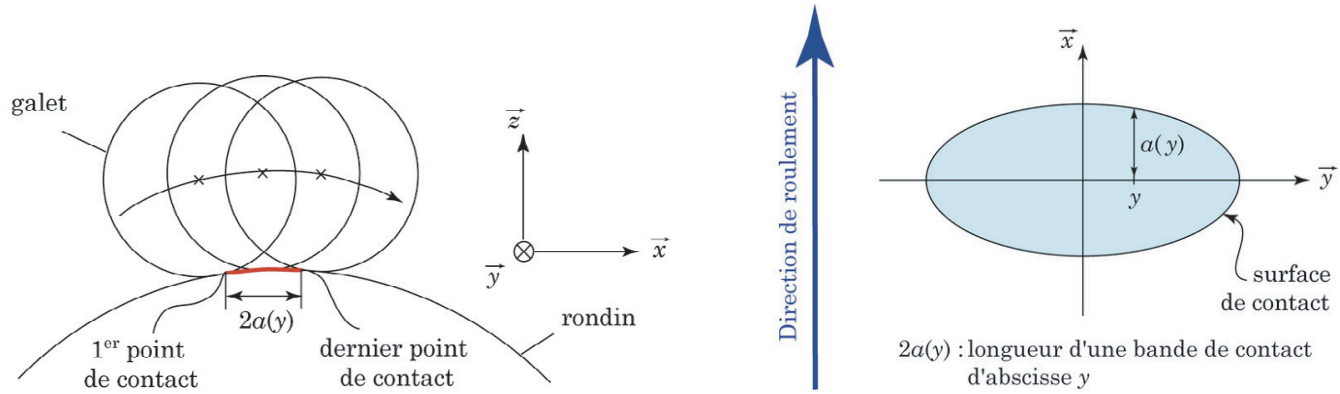

Fig. 16. Surface de contact et définition de la longueur de contact $2 a(y)$.

Sur la surface élémentaire $\mathrm{d} S$, la relation précédente devient :

$$
\frac{\mathrm{d} \dot{W}}{\mathrm{~d} S} p(x, y) \omega_{\mathrm{g}}(x, y) \text { avec } p(x, y)=\frac{\mathrm{d} F}{\mathrm{~d} S}
$$

L'usure provient donc du glissement au niveau de la surface de contact. Dans la zone où $w_{\mathrm{g}}$ n'est pas nulle, la loi de frottement de Coulomb lie la pression et la force tangentielle surfacique $(\tau=\mu p)$ où $\mu$ est le coefficient de frottement.

$$
\dot{u}(x, y)=\frac{\mathrm{d} \dot{W}}{\mathrm{~d} S}=\frac{K^{\prime}}{H} \tau(x, y) w_{\mathrm{g}}(x, y) \text { avec } K=\mu K^{\prime}
$$

Le taux de profondeur d'usure apparaît comme proportionnel à la puissance dissipée surfacique. L'incrément d'usure $u$ généré suite au passage d'un galet est obtenu en intégrant le taux de profondeur usée sur la durée durant laquelle un point du rondin « voit » passer les points du galet comme illustré sur la figure 16.

$$
u(y)=\frac{K^{\prime}}{H V} \int_{-a(y)}^{a(y)} \tau(x, y) \omega_{\mathrm{g}}(x, y) \mathrm{d} x=\frac{K^{\prime}}{H V} P_{1}(y)
$$

$P_{1}(y)$ est la puissance dissipée linéique $\left[\mathrm{W} \cdot \mathrm{m}^{-1}\right]$. Pour mener à bien ce calcul, il est nécessaire de connaître les forces surfaciques tangentielles $\tau$ et les vitesses de glissement $w_{\mathrm{g}}$ en tous points de la surface de contact. Il faut donc, au préalable, résoudre le problème du contact roulant stationnaire entre deux solides de révolution quelconques.

\subsection{Résolution du problème de contact}

Boussinesq en 1885 [19], à partir de la théorie de l'élasticité, est le premier à résoudre le cas de contact surfacique entre solides élastiques. Il établit des relations « intégrales » entre la différence de déplacement élastique en un point du contact, la répartition de pression $p$ et les forces surfaciques tangentielles $\tau_{x}$ et $\tau_{y}$. Lorsque les corpsen contact ont les mêmes propriétés élastiques, alors le problème normal est découplé du problème tangent et les relations de comportement ont la forme suivante :

$$
\begin{aligned}
u_{x}(x, y)= & \frac{1}{\pi G} \iint_{\text {contact }}\left(\left\{\frac{1-v}{r}+\frac{v\left(x-x^{\prime}\right)^{2}}{r^{3}}\right\} \tau_{x}\left(x^{\prime}, y^{\prime}\right)\right. \\
& \left.+\frac{v\left(x-x^{\prime}\right)\left(y-y^{\prime}\right)}{r^{3}} \tau_{y}\left(x^{\prime}, y^{\prime}\right)\right) \mathrm{d} x^{\prime} \mathrm{d} y^{\prime} \\
u_{y}(x, y)= & \frac{1}{\pi G} \iint_{\text {contact }}\left(\frac{v\left(x-x^{\prime}\right)\left(y-y^{\prime}\right)}{r^{3}} \tau_{x}\left(x^{\prime}, y^{\prime}\right)\right. \\
& \left.+\left\{\frac{1-v}{r}+\frac{v\left(y-y^{\prime}\right)^{2}}{r^{3}}\right\} \tau_{y}\left(x^{\prime}, y^{\prime}\right)\right) \mathrm{d} x^{\prime} \mathrm{d} y^{\prime} \\
u_{z}(x, y)= & \frac{1-v}{\pi G} \iint_{\text {contact }} \frac{p\left(x^{\prime}, y^{\prime}\right)}{r} \mathrm{~d} x^{\prime} \mathrm{d} y^{\prime} \\
\text { avec } r= & \sqrt{\left(x-x^{\prime}\right)^{2}+\left(y-y^{\prime}\right)^{2}}
\end{aligned}
$$

$u_{x}(x, y), u_{y}(x, y), u_{z}(x, y)$ sont les composantes du déplacement relatif entre un solide 1 et un solide 2 dans les directions $\vec{x}, \vec{y}$ et $\vec{z}$. Le module de rigidité de cisaillement $G$ et le coefficient de Poisson $v$ sont définis par :

$$
\frac{1}{G}=\frac{1}{2 G_{1}}+\frac{1}{2 G_{2}} ; \quad \frac{v}{G}=\frac{v_{1}}{2 G_{1}}+\frac{v_{2}}{2 G_{2}}
$$

Les travaux de Vermeulen et de Johnson [20,21] sont une extension tridimensionnelle des résultats de Carter [22]. Ils traitent du problème de contact surfacique d'une bille roulant sur un plan. Aux relations précédentes s'ajoutent les conditions de non interpénétration des solides, la loi de frottement ( $\mu$ est le coefficient de frottement) et les relations cinématiques de roulement :

$$
\left\{\begin{array}{l}
\text { Condition d'adhérence : } \\
\vec{\omega}_{\mathrm{g}}(x, y)=\overrightarrow{0} \rightarrow|\vec{\tau}(x, y)|<\mu p(x, y) \\
\text { Condition de glissement : } \\
\vec{\omega}_{\mathrm{g}}(x, y) \neq \overrightarrow{0} \rightarrow|\vec{\tau}(x, y)|<\mu p(x, y) \\
\text { et } \vec{\tau}(x, y)=-\frac{\mu p(x, y)}{\left|\vec{\omega}_{\mathrm{g}}(x, y)\right|} \vec{\omega}_{\mathrm{g}}(x, y)
\end{array}\right.
$$

Le rondin cylindrique de rayon $R_{\mathrm{r}}$ (Fig. 17) est entraîné en rotation à une vitesse $\omega_{\mathrm{r}}$. Le galet est de rayon $R_{\mathrm{g}}$ et présente un rayon de bombé $R_{\mathrm{b}}$. Dans ces conditions le contact est quasi-ponctuel et reste ponctuel même si les 

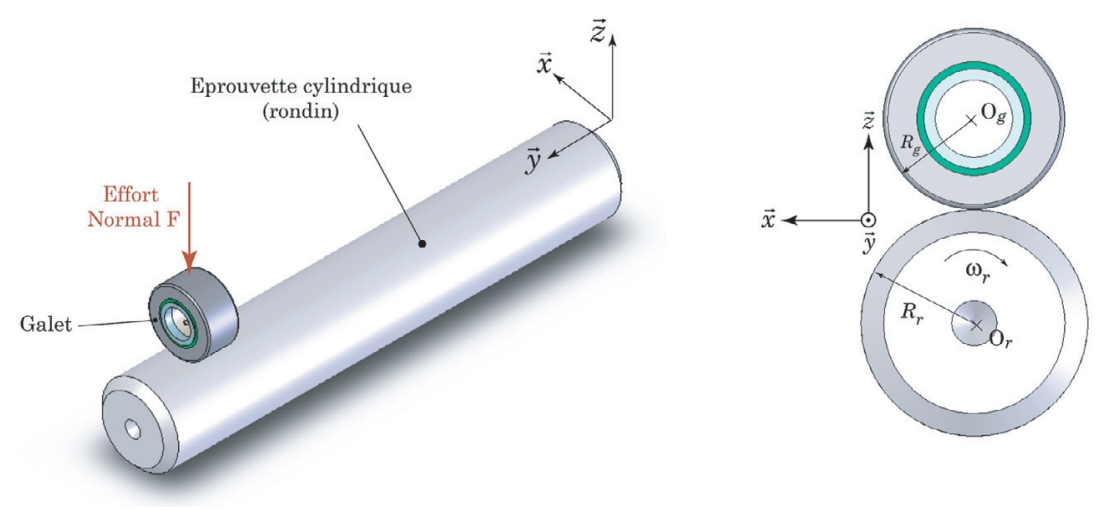

Fig. 17. Description géométrique du contact roulant entre le galet et le rondin.

axes de rotations de ces deux solides ne sont pas parfaitement parallèles. La vitesse de renouvellement du contact est portée par l'axe $\vec{x}$ et l'axe de rotation du rondin définit l'axe $\vec{y}$.

L'axe $\vec{z}$ est normal au contact. Les déformations sont très localisées au voisinage du contact, nous pouvons considérer que la vitesse de glissement peut être obtenue par superposition de la vitesse de glissement de corps rigides et de la contribution des déformations élastiques des solides en contact. La partie « rigide» de la vitesse de glissement en tous points de la zone de contact est donnée par les relations :

$$
\left\{\begin{array}{l}
V_{\mathrm{g} x}=R_{\mathrm{r}} \omega_{\mathrm{r}}+R_{\mathrm{g}} \omega_{\mathrm{g}}+y \varphi_{x} \omega_{\mathrm{g}} \\
V_{\mathrm{g} x}=R_{\mathrm{g}} \varphi_{z} \omega_{\mathrm{g}}-x \varphi_{x} \omega_{\mathrm{g}}
\end{array}\right.
$$

Pour travailler avec des grandeurs sans dimensions, on divise les relations du système précédent par la vitesse de renouvellement du point de contact $V$ (qui vaut $R_{\mathrm{r}} \omega_{\mathrm{r}}$ ou environ $\left.-R_{\mathrm{g}} \omega_{\mathrm{g}}\right)$; ce qui donne :

$$
\left\{\begin{array}{l}
\frac{V_{\mathrm{g} x}}{V}=v_{x}-y \phi \\
\frac{V_{\mathrm{g} y}}{V}=v_{y}-x \phi
\end{array} \quad \text { avec }: v_{x} \frac{\delta V_{x}}{V} v_{y} \frac{\delta V_{y}}{V}=\varphi_{z} \phi=\frac{\varphi_{x}}{R_{\mathrm{g}}}\right.
$$

Les trois quantités $v_{x}, v_{y}$ et $\phi$ sont appelées des pseudoglissements, et ils influencent grandement la puissance dissipée au niveau du contact. On les appelle respectivement pseudoglissement longitudinal (ou de traînée), pseudoglissement transversal (ou de dérive) et pseudoglissement de spin (ou de pivotement).

Soient $u_{x}$ et $u_{y}$ les composantes des déplacements relatifs entre le galet et le rondin provenant des déformations. Ces déplacements contribuent aussi à la vitesse de glissement locale. En effet, la dérivée particulaire du déplacement provenant des déformations s'écrit :

$$
\frac{\mathrm{d} \vec{u}}{\mathrm{~d} t}=\frac{\partial \vec{u}}{\partial t}+\frac{\partial \vec{u}}{\partial x} \frac{\mathrm{d} x}{\mathrm{~d} t}+\frac{\partial \vec{u}}{\partial y} \frac{\mathrm{d} y}{\mathrm{~d} t}+\frac{\partial \vec{u}}{\partial z} \frac{\mathrm{d} z}{\mathrm{~d} t}=\frac{\partial \vec{u}}{\partial t}-\underline{\operatorname{grad}} \vec{u} \cdot \vec{V}
$$

Le point $M$ de la zone de contact en $x, y, z$ « voit » passer les corps à la vitesse de renouvellement du contact dirigée suivant $\vec{x}$ : sa vitesse par rapport à la zone de contact est donc opposée ce qui justifie le signe $«-»$ devant le gradient. Dans le cas stationnaire le système complet des équations cinématiques devient :

$$
\left\{\begin{array}{l}
\frac{\omega_{\mathrm{g} x}}{V}=v_{x}-y \phi-\frac{\partial u_{x}}{\partial x} \\
\frac{\omega_{\mathrm{g} y}}{V}=v_{y}-x \phi-\frac{\partial u_{y}}{\partial x}
\end{array}\right.
$$

Kalker [23] propose de discrétiser l'aire potentielle de contact et résout le problème numériquement. Cette résolution, dite « exacte », fait l'objet d'un logiciel commercialisé sous le nom de «CONTACT » [24]. L'utilisation de ce logiciel pour la simulation de l'usure n'a pas été retenue car elle nécessite des temps de calculs importants et non adaptés, ni à l'approche itérative pour faire évoluer les profils en fonction de l'usure; ni à l'approche probabiliste présentée plus loin. Nous retenons la théorie simplifiée du contact roulant dans laquelle on suppose la linéarité entre les déplacements relatifs et les force surfaciques tangentielles (hypothèse «tapis de ressorts »). Les rigidités associées à ces ressorts dépendent de la géométrie des corps en contact et Kalker propose de les identifier en comparant cette approche à sa théorie linéaire; ce qui aboutit au système à résoudre suivant :

$$
\left\{\begin{array}{l}
\frac{\omega_{\mathrm{g} x}}{V L}=\frac{V_{x}}{L_{1}}-\frac{\phi y}{L_{3}}-\frac{\partial \tau_{x}}{\partial x} \\
\frac{\omega_{\mathrm{g} y}}{V L}=\frac{V_{y}}{L_{2}}-\frac{\phi x}{L_{3}}-\frac{\partial \tau_{y}}{\partial x}
\end{array}\right.
$$

avec les flexibilités $L_{i}$ qui dépendent des coefficients $C_{i j}$ eux-mêmes définis par des intégrales sur l'ellipse de contact et tabulés comme les coefficients $m, n$ et $r$ de Hertz en fonction de l'élancement de l'ellipse de contact $b / a$ et du coefficient de Poisson équivalent $v$ :

$$
L_{1}=L_{x}=\frac{8 a}{3 C_{11} G}, L_{2}=L_{y}=\frac{8 a}{3 C_{22} G}, L_{3}=\frac{\pi a \sqrt{a / b}}{4 C_{23} G}
$$

$L$ est la flexibilité équivalente. C'est une moyenne pondérée par les pseudoglissements. Aucune justification théorique n'est associée à l'expression de cette flexibilité équivalente :

$$
L=\frac{v_{x} L_{1}+v_{y} L_{2}+\sqrt{a b} \phi L_{3}}{\sqrt{v_{x}^{2}+v_{y}^{2}+a b \phi^{2}}}
$$


L'algorithme FASTSIM [25] permet de résoudre le problème ainsi simplifié. Des comparaisons entre FASTSIM et CONTACT montrent une bonne approximation de la solution exacte par la solution approchée [10,26].

L'usure générée à chaque pas de temps lors de la simulation fait évoluer les courbures localement. Dans ces conditions, même si la géométrie initiale satisfait les hypothèses de Hertz, assez rapidement l'usure modifie la géométrie et le problème normal doit être résolu par une méthode adaptée aux contacts non-hertziens. La démarche que nous avons choisie de retenir est appelée méthode semi-hertzienne par Ayasse et Chollet [27], elle est proche des travaux de Kik et Piotrowski [28]. La zone de contact est découpée en bandes (indicées $« i »$ ) et la résolution du problème normal et ensuite du problème tangent est effectuée en appliquant l'algorithme FASTSIM par bande.

L'approche simplifiée consiste à estimer la zone de contact et la pression de contact en se basant sur la pénétration des corps à l'état non déformé : c'est la résolution du problème normal. Cette approche est évidemment fausse dans le principe puisqu'elle ne redonne ni la taille, ni l'élancement de l'ellipse de Hertz lorsque les courbures sont constantes. Une correction est donc opérée pour retrouver les résultats de Hertz.

Par la suite, toutes les notations indicées $« i$ seront définies pour une bande de contact $« y=y_{i} »$. De même le « $»$ indique l'éprouvette cylindrique (rondin) et le $« \mathrm{~g} »$ indique le galet. Les relations établies pour un contact de Hertz sont étendues et calculées pour chaque bande de contact $\ll i »$. Ainsi :

$$
\begin{aligned}
& A=\frac{1}{2}\left\{\frac{1}{R_{y \mathrm{e}}}-\frac{1}{R_{y \mathrm{~g}}}\right\} \rightarrow A_{i}=\frac{1}{2}\left\{\frac{1}{R_{y \mathrm{e}}^{i}}-\frac{1}{R_{y \mathrm{~g}}^{i}}\right\}, \\
& B=\frac{1}{2}\left\{\frac{1}{R_{y \mathrm{e}}}-\frac{1}{R_{y \mathrm{~g}}}\right\} \rightarrow B_{i}=\frac{1}{2}\left\{\frac{1}{R_{x \mathrm{e}}^{i}}-\frac{1}{R_{x \mathrm{~g}}^{i}}\right\} \text {, } \\
& \text { et } \lambda_{i}=\frac{A_{i}}{B_{i}}
\end{aligned}
$$

Les indices $« x »$ et $« y »$ indiquent que les rayons de courbures sont définis autour des axes $x$ et $y$. Après correction, la géométrie de la surface de contact est décrite par :

$$
a_{i}=\sqrt{\frac{h_{i}}{A_{\mathrm{c} i}}} \text { avec } h_{i}=z_{\mathrm{g}}-z_{\mathrm{e}}
$$

où les $A_{\mathrm{c} i}$ sont les courbures corrigées, les $a_{i}$ et $h_{i}$ sont définis sur la figure 18.

Les raideurs normales de chaque bande de contact $K_{i}$ sont définies à partir des formules de Hertz.

$$
K_{i}=\frac{E}{2\left(1-v^{2}\right)} \cdot \frac{1+\lambda_{i}}{n_{i}^{3}} \cdot \Delta y
$$

En considérant une répartition elliptique de chargement dans la direction de roulement, nous exprimons la répartition de pression à une abscisse $y=y_{i}$ :

$$
p\left(x, y_{i}\right)=\frac{2 \Delta N_{i}}{\pi a_{i} \Delta y} \sqrt{1-\frac{x^{2}}{a_{i}^{2}}}
$$

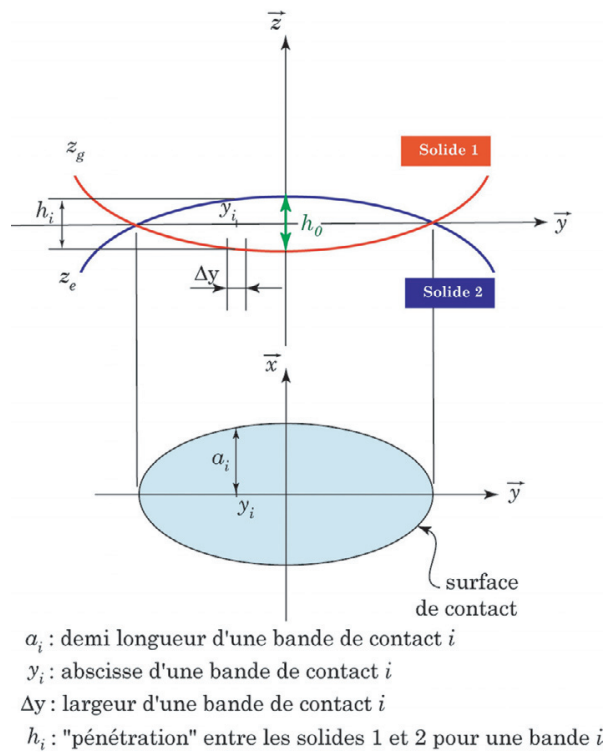

Fig. 18. Définition du géométrique de l'interpénétration pour la résolution du problème normal.

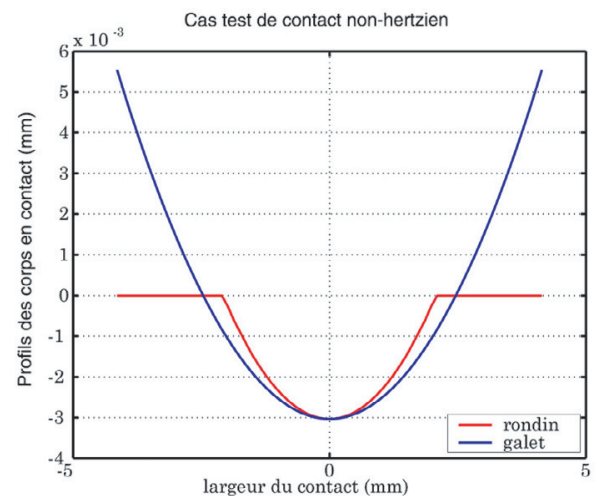

Fig. 19. Cas test d'un contact non-hertzien.

$\Delta N_{i}$ est la contribution d'une bande $« i »$ de largeur $\Delta y$ et d'abscisse $y_{i}$, dans la charge normale globale $F$. Cette charge $\Delta N_{i}$ s'exprime en fonction de la pénétration $h_{i}$ :

$$
\Delta N_{i}=K_{i} h_{i}
$$

La valeur de l'interpénétration $h_{0}$ est ajustée jusqu'à vérifier le critère :

$$
\sum_{i} \Delta N_{i}=F
$$

Dans [29] pour le contact came-galet et dans [27,30] pour le contact roue-rail, les auteurs montrent que pour obtenir une bonne corrélation avec les résultats du logiciel CONTACT ou les résultats d'une modélisation par la méthode des éléments-finis, il est nécessaire de lisser ou diffuser les courbures. La méthode de diffusion choisie est la méthode des éléments diffus [31]. Elle a aussi fait l'objet d'une présentation dans [29].

La résolution du problème tangent est ensuite identique à l'algorithme FASTSIM pourvu que les $C_{i j}$ et $L_{i}$ 

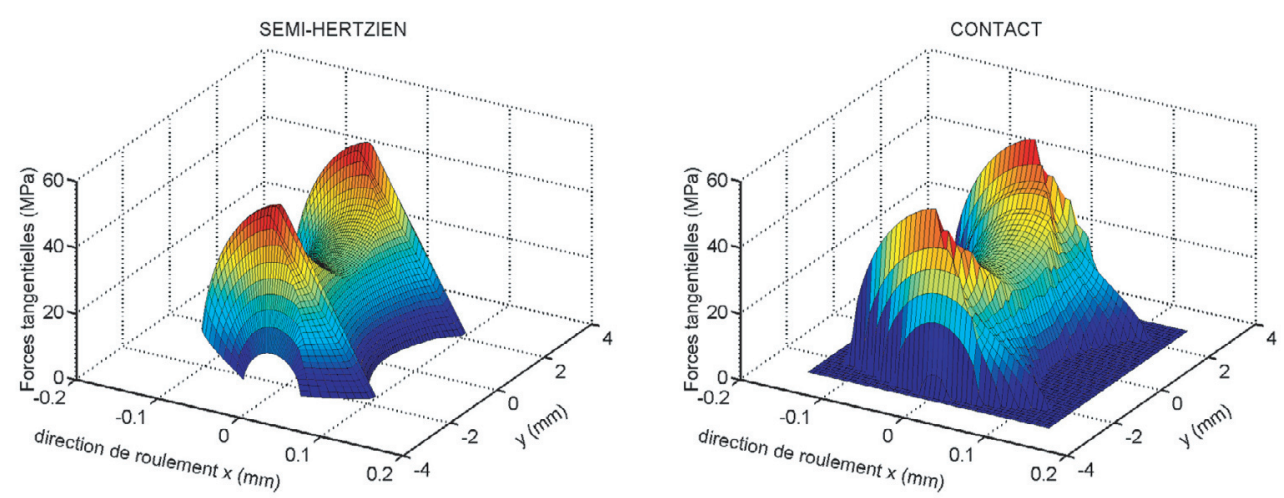

Fig. 20. Distribution des forces tangentielles par CONTACT et l'approche semi-hertzienne.
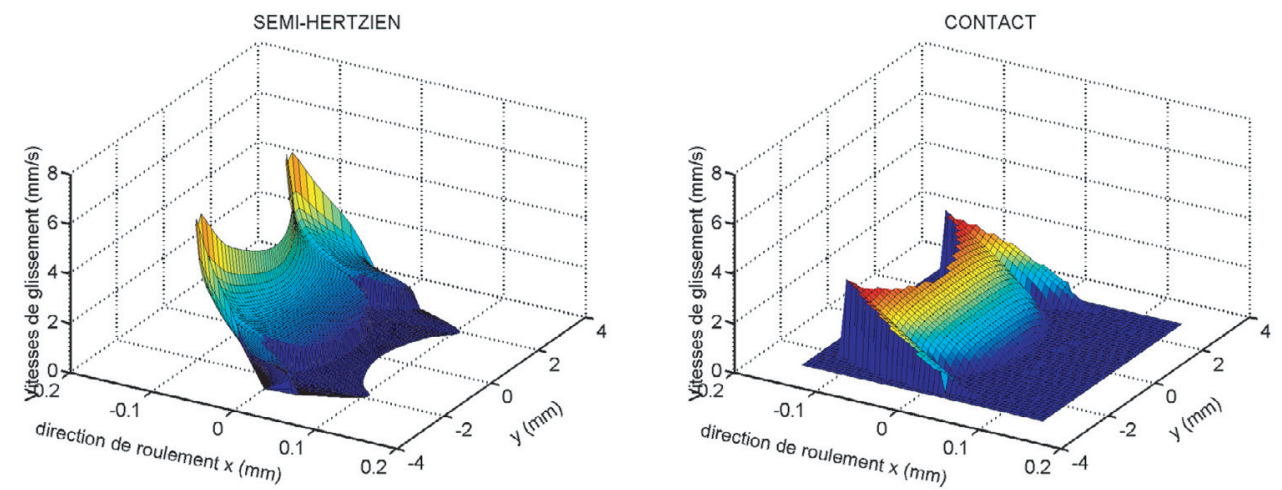

Fig. 21. Distributions des vitesses de glissement par CONTACT et l'approche semi-hertzienne.

soient définis pour chaque bande $y_{i}$. Pour valider l'utilisation de l'algorithme FASTSIM par bande dans le cas de contact non-hertzien, nous allons considérer le cas test présenté sur la figure 19 et confronter ces résultats aux résultats obtenus avec l'approche dite « exacte »du logiciel CONTACT. Le rayon de courbure du rondin varie fortement et ce cas est représentatif d'un calcul à traiter après « usure »du rondin.

Les figures 20 et 21 présentent les résultats obtenus lors de la résolution du problème tangent par la méthode semi-hertzienne couplée à l'algorithme FASTSIM et par le logiciel CONTACT. Ces résultats sont les distributions de la vitesse de glissement et des forces surfaciques tangentielles. Nous pouvons noter l'approximation linéaire faite avec la méthode semi-hertzienne sur la répartition des contraintes tangentielles de la figure 20. Cependant, en moyenne, les deux distributions sont identiques.

Nous pouvons constater également que la partition adhérence/glissement est proche. Nous retrouvons l'asymptote verticale (la vitesse de glissement n'est pas définie sur le bord de fuite du contact) observée dans FASTSIM. Ce résultat n'est pas pénalisant sur l'usure dans la mesure où la puissance dissipée reste finie. De plus, c'est la puissance dissipée linéique qui intervient dans le calcul de l'usure, nous devons comparer le calcul de cette puissance par CONTACT et par semi-hertzien. La figure 22 présente cette comparaison.

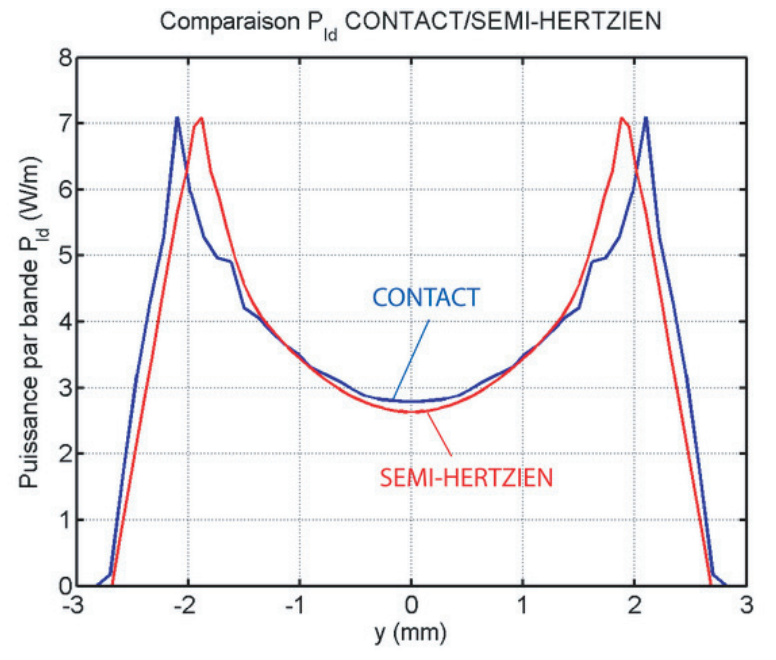

Fig. 22. Puissance dissipée linéique obtenue par CONTACT et par semi-hertzienne.

Nous pouvons constater que les pics observés sur la puissance dissipée linéique sont décalés de quelques dixièmes de millimètres. En calculant la puissance dissipée totale, l'écart entre les deux approches est de $1 \%$. Ce résultat est très satisfaisant si l'on considère le gain en temps de calcul : CONTACT (en fortran 77) est 1700 fois plus lent que SEMI-HERTZIEN (en Matlab). Les calculs 


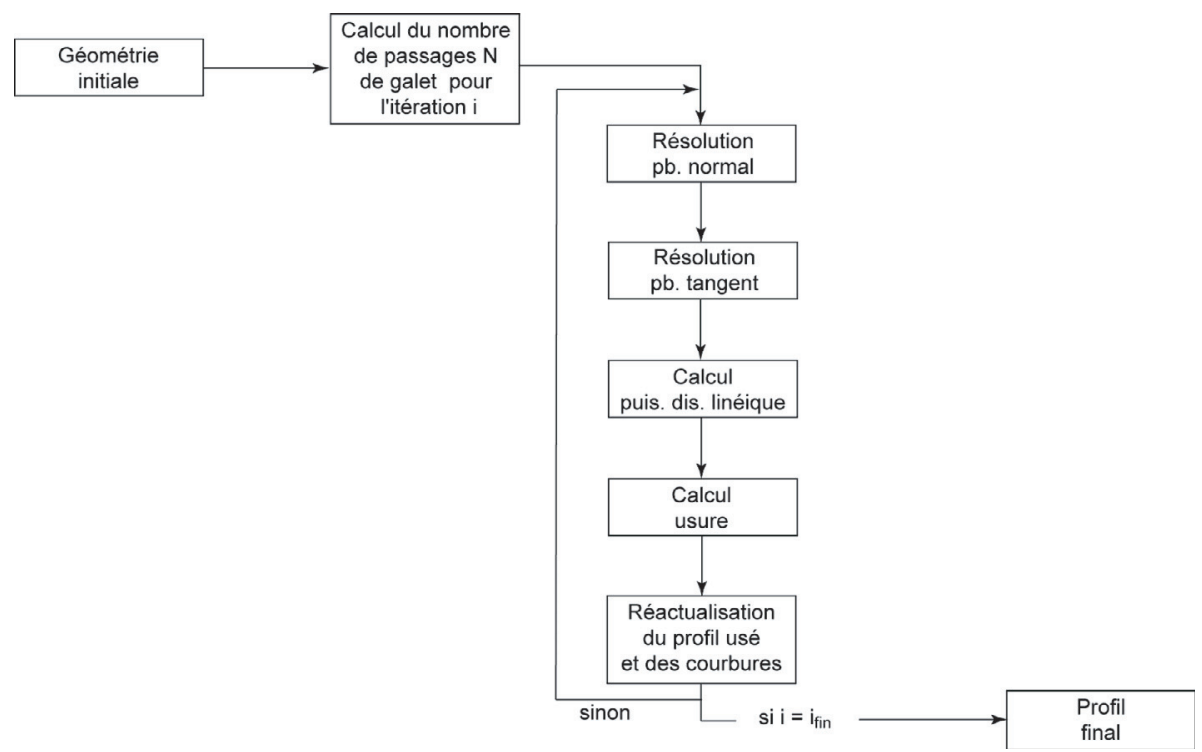

Fig. 23. Démarche de résolution du problème d'usure.

ont été effectués sur la même machine et pour la même discrétisation du problème (grille de $50 \times 50$ ).

\subsection{Démarche de résolution du problème d'usure}

La résolution du problème d'usure se décompose en plusieurs étapes présentées sur la figure 23. A priori, une itération $« i$ équivaut à un passage de galet. Mais l'usure ainsi calculée est très faible et ne modifie que très peu la géométrie.

Nous pouvons accélérer la simulation en supposant que l'évolution de l'usure est linéaire en fonction du nombre de passages du galet tant que cette usure ne modifie pas le profil de manière significative. Pour diminuer la durée de simulation, à chaque itération $\langle i »$ on considère qu'il y a $N$ passages de galet. Sur le banc d'essai, un tour de rondin équivaut à trois passages de galet. La relation qui lie la durée de l'essai $T$, le nombre d'itérations $I$, la vitesse de rotation du rondin $\omega_{\mathrm{r}}$ et le nombre $N$ de passages du galet est :

$$
T=\frac{I N}{3 \omega_{\mathrm{r}}}
$$

Le nombre d'itérations $I$ dépend du coefficient d'usure $K^{\prime}$. En effet si ce coefficient est important, l'usure augmentera rapidement et le nombre $N$ de passages par itération doit être faible. En effet, la largeur du contact (dans la direction $y$ ) est fixée par la résolution du problème normal et la profondeur d'usure est liée à la puissance dissipée, au coefficient d'usure et au nombre de passages du galet par :

$$
u(y)=K N P_{1}(y)
$$

Dans le cas d'une distribution de puissance dissipée linéique symétrique, la puissance est maximale au centre du profil. Dans ce cas l'usure maximale vaut :

$$
u_{\max }=u\left(y_{\mathrm{c}}\right)=K N P_{10} \text { pour } P_{10}=P_{1}\left(y_{\mathrm{c}}\right)
$$

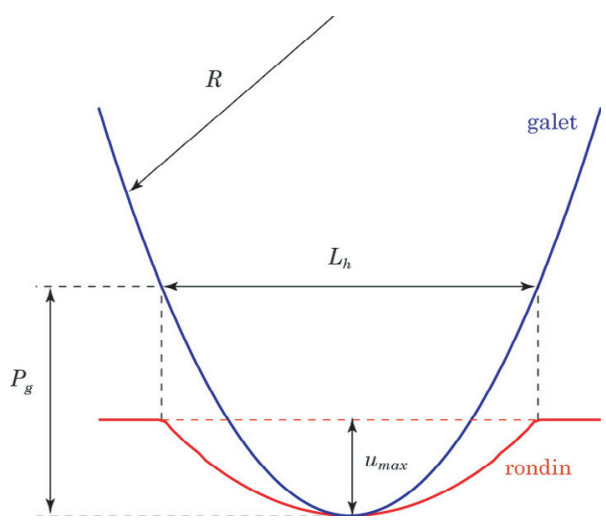

Fig. 24. Définition du paramètre $D$.

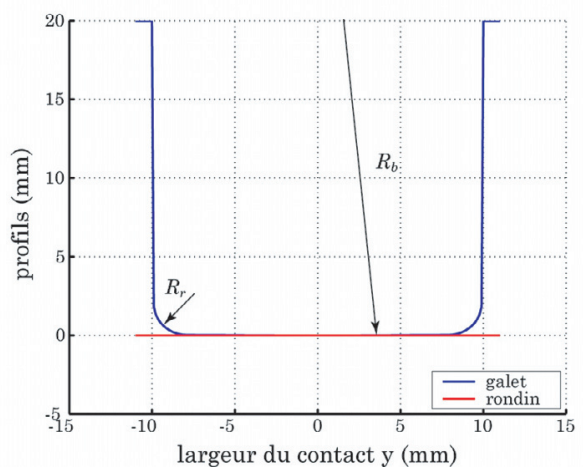

Fig. 25. Définition des géométries initiales du galet et de l'éprouvette.

où $y_{\mathrm{c}}$ est l'abscisse du centre du profil. Proche du point de contact, le profil du galet est circulaire comme présenté sur la figure 24 . On définit le paramètre $D$ par :

$$
D=\frac{P_{\mathrm{g}}}{u_{\max }}
$$



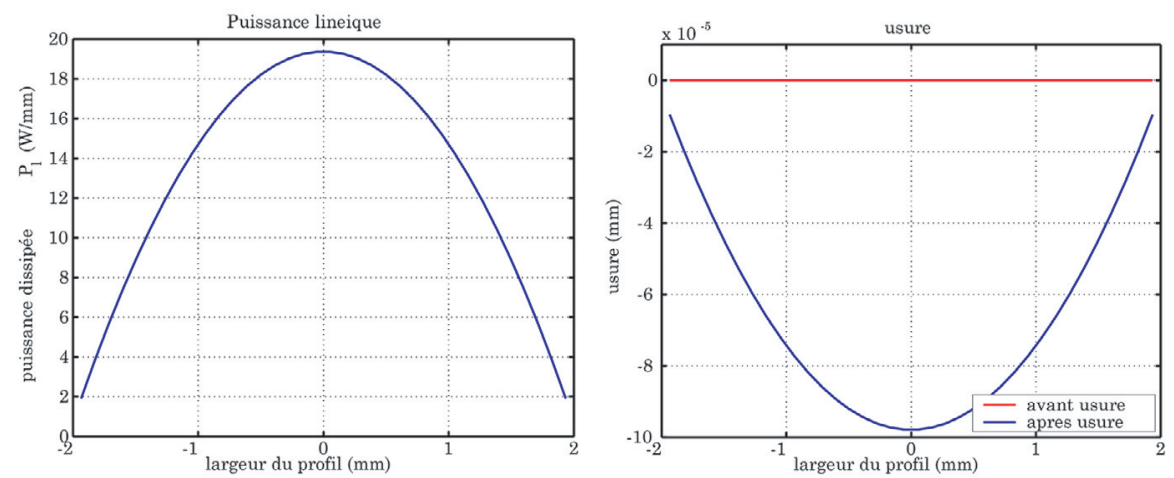

Fig. 26. Puissance dissipée linéique et usure du rondin.

En combinant les relations précédentes on peut évaluer le nombre de passages $N$ de galet par itération :

$$
N=\frac{P_{\mathrm{g}}}{D K P_{10}}
$$

$P_{\mathrm{g}}$ est la hauteur du profil du galet pour la largeur $L_{\mathrm{h}}$ du profil usé comme présenté figure 24. Le paramètre $D$ permet de contrôler la vitesse d'avance de l'usure par itération. Ce calcul est effectué dans le cas du contact hertzien de la première itération de la simulation de l'usure de manière à fixer le nombre de passages du galet $N$ et par suite le nombre d'itérations $I$ nécessaires pour atteindre la durée $T$ souhaitée. À partir d'essais numériques on constate que $D$ doit être plus grand que 1 . Cela suppose que l'empreinte d'usure laissée par le passage du galet ne peut pas être plus profonde et surtout moins large que la géométrie du galet. Plus $D$ est grand et plus le nombre de passages $N$ de galet par itération est faible et plus le nombre d'itérations $I$ est important. Il faut donc fixer $D$ pour avoir le meilleur rapport précision/temps de calcul; $D=1,1$ est un compromis raisonnable entre stabilité du calcul et rapidité.

Les solides en contact sont de révolution; ainsi, l'usure sera axisymétrique. Le problème peut donc se simplifier à l'étude des profils du galet et du rondin en contact au fur et à mesure des itérations. Les profils du galet et du rondin avant usure sont présentés figure 25 .

La puissance dissipée linéique est tracée sur la figure 26 ainsi que l'usure correspondante. Seule la partie en contact est représentée. Pour cet exemple nous avons retenu une valeur arbitraire de 0,01 pour $K^{\prime}$ en présence de pseudoglissement longitudinal uniquement. Nous verrons par la suite une méthode d'identification pour déterminer ce coefficient d'usure. Le rayon de courbure du rondin avant usure est infini dans le plan du profil.

L'usure «creuse » le profil du rondin et celui-ci est actualisé à l'étape suivante. Les courbures évoluent et il faut les calculer à chaque itération : cela présente une difficulté numérique. Pour expliquer cette difficulté, nous allons considérer le cas suivant : le profil du rondin $z_{\mathrm{e}}$ obtenu après un certain nombre de passages du galet. Ce profil, présenté sur la figure 27, peut se découper en trois zones :

$-1^{\text {re }}$ partie : le profil est rectiligne $z_{1}=0$;

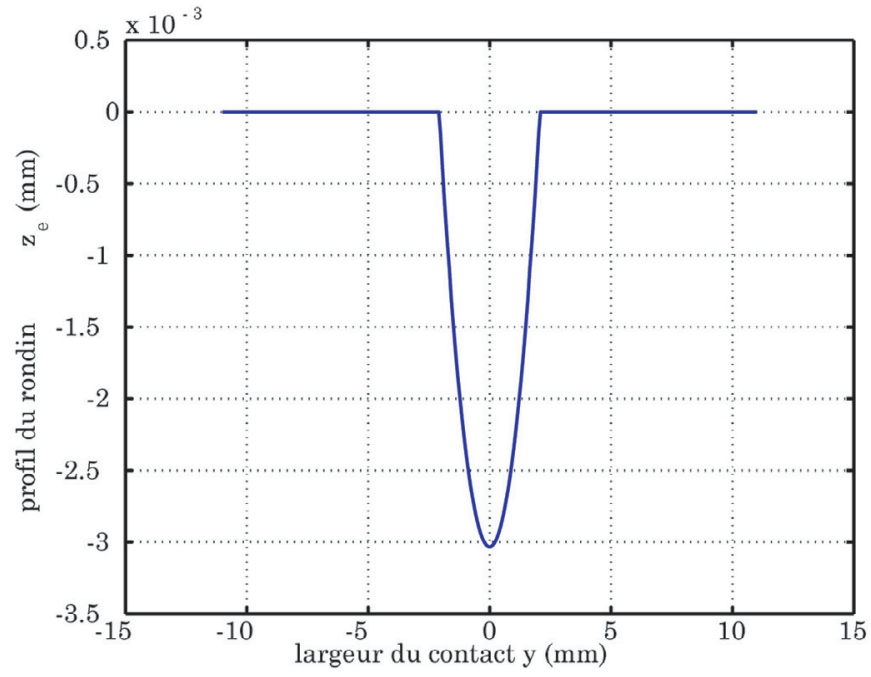

Fig. 27. Profil usé du rondin utilisé comme exemple.

$-2^{\mathrm{e}}$ partie : le profil est assimilé à une parabole $z_{2}=$ $a y^{2}+c$

$-3^{\mathrm{e}}$ partie : le profil est à nouveau rectiligne $z_{3}=z_{1}=$ 0 .

Les jonctions entre la $1^{\mathrm{re}}-2^{\mathrm{e}}$ partie et $2^{\mathrm{e}}-3^{\mathrm{e}}$ partie présentent des points anguleux : soit une discontinuité du rayon de courbure. Ces discontinuités sont pénalisantes car elles vont propager des fluctuations au niveau de la forme de l'aire de contact, des efforts par bandes et de la puissance dissipée linéique. Au fur et à mesure de la progression de l'usure, ces fluctuations vont apparaître sur le profil usé jusqu'à faire diverger le calcul.

Afin d'éliminer ces points anguleux on va émousser les angles par une fonction de type «tangente hyperbolique . Considérons les trois parties du profil définies au début de ce paragraphe et posons :

$$
\psi(y)=\tanh \left(\alpha\left(y-y_{0}\right)\right)
$$

où $\alpha$ et $y_{0}$ sont des paramètres à régler pour rendre la méthode efficace. Le profil du rondin étant symétrique, nous ne considérons que la moitié du profil et définissons la fonction $z$ par :

$$
z(y)=\frac{1}{2}\left((1-\psi(y)) z_{1}(y)+(1+\psi(y)) z_{2}(y)\right)
$$



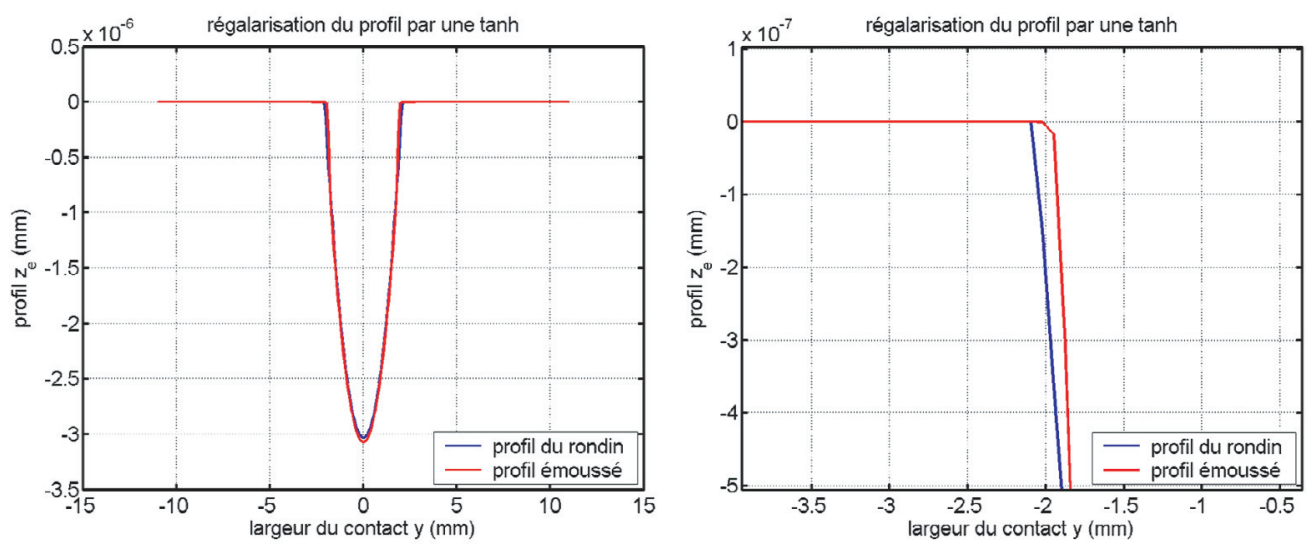

Fig. 28. Approximation du profil usé du rondin par une fonction permettant d'émousser les points anguleux.

Cette fonction permet de régulariser le profil $z_{\mathrm{e}}$ comme présenté sur la figure 28. Nous pouvons constater que la surface d'usure est conservée entre le profil usé et le profil régularisé. Définissons l'abscisse du point anguleux du rondin par $y_{\mathrm{r} 1}$. Pour les valeurs de $y$ très inférieures à $y_{\mathrm{r} 1}$ la fonction $z$ vaut $z_{1}$ et pour les valeurs de $y$ très supérieures à $y_{\mathrm{r} 1}$ la fonction $z$ vaut $z_{2}$. $y_{0}$ doit donc être proche de $y_{\mathrm{r} 1}$ et $\alpha$ suffisamment «petit» pour ne pas « trop » émousser l'angle.

L'approximation du profil du rondin $z_{\mathrm{e}}$ par $z$ permet de définir une fonction continue et dérivable. Les dérivées sont définies analytiquement ainsi que les rayons de courbures et les fortes discontinuités au niveau des points anguleux disparaissent rendant la méthode itérative plus stable.

\subsection{Simulation d'usure : banc d'essai à galets}

Pour illustrer la faisabilité de la simulation on retient le jeu de paramètres suivants : $\mu=0,08, F=300 \mathrm{~N}$, $K^{\prime}=0,01$ et $v_{x}=0,065 \%$. Ces valeurs ne sont pas issues de l'identification mais sont néanmoins réalistes. Le coefficient de stabilité numérique $D$ est fixé à 1,1 . Les calculs ont été effectués en considérant du glissement uniquement dans la direction de roulement : les pseudoglissements de spin et de dérive sont nuls.

La figure 29 présente l'évolution de l'usure maximale en fonction de la durée de l'essai. Après une phase non-linéaire (entre 0 et $0,5 \times 10^{6}$ cycles), l'usure croît linéairement en fonction du temps. Ce résultat s'explique par le fait qu'au-delà des $0,5 \times 10^{6}$ premiers cycles le contact entre le galet et le rondin devient conforme (Fig. 30) et la pression de contact est uniforme dans la direction orthogonale à la direction de roulement. Dans ces conditions, la puissance dissipée linéique est uniforme ainsi que l'usure. Ce résultat est intéressant car il montre que le modèle mécanique rend compte du comportement non-linéaire observé lors des essais d'usure.

L'usure générée sur les bords du contact suit les rayons de raccordement du galet. Cette forme de profil est observée expérimentalement. Un avantage de cette

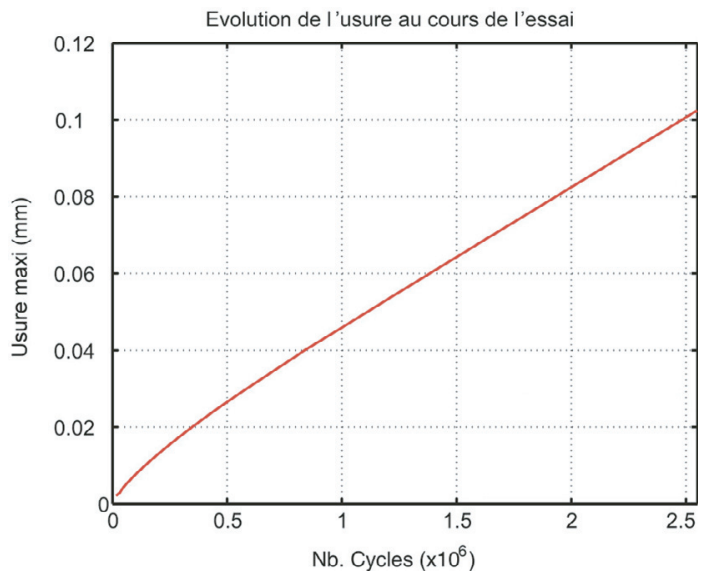

Fig. 29. Évolution de l'usure maximale simulée en fonction de la durée de l'essai.

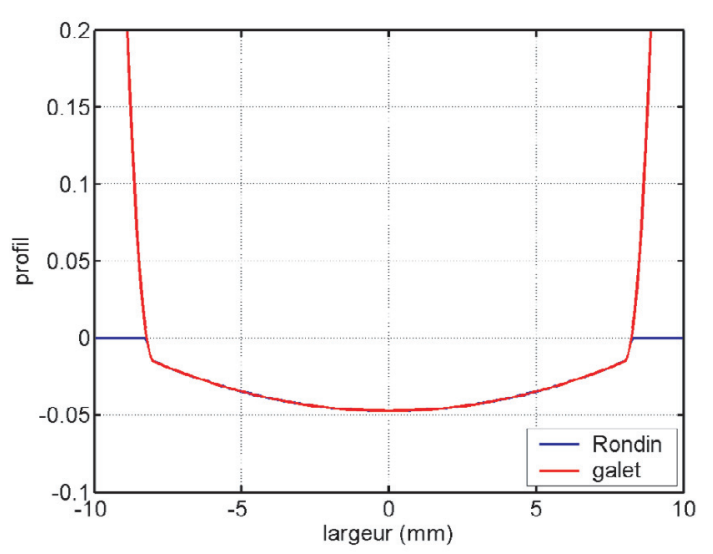

Fig. 30. Profil du rondin en fin de simulation.

modélisation est que l'on peut suivre l'évolution des profils au cours de la simulation. Ces profils peuvent être comparés aux profils mesurés expérimentalement. 


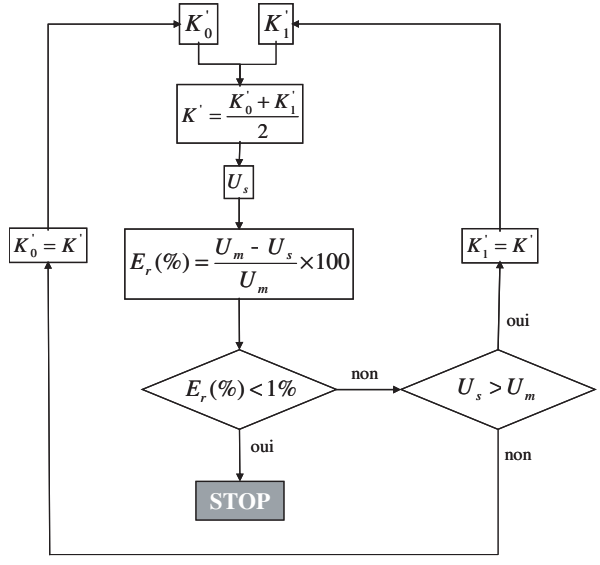

Fig. 31. Algorithme d'identification de la loi d'usure.

\subsection{Identification du coefficient d'usure}

Nous ne pouvons pas identifier notre modèle d'usure sur le banc d'essai à galets car les dispersions sont trop nombreuses. L'estimation du coefficient d'usure $K^{\prime}$ est obtenue grâce aux essais réalisés sur le banc d'essai bidisques. Cette identification est effectuée par dichotomie : deux coefficients $K_{0}^{\prime}$ et $K_{1}^{\prime}$ sont choisis arbitrairement mais ils doivent englober la solution cherchée. $K^{\prime}$ sera la moyenne des coefficients $K_{0}^{\prime}$ et $K_{1}^{\prime}$. Le principe de la méthode est de réduire l'intervalle $\left[\begin{array}{lll}K_{0}^{\prime} & K_{1}^{\prime}\end{array}\right]$ pour converger vers $K_{0}^{\prime}=K_{1}^{\prime}=K^{\prime}$. À chaque étape de la procédure une simulation d'usure est effectuée : un profil d'usure simulé $u_{\mathrm{S}}(y)$ est obtenu. L'usure maximale $U_{\mathrm{S}}$ (le maximum de la profondeur usée) est comparée à la valeur d'usure maximale expérimentale $U_{\mathrm{m}}$. Un écart relatif $E_{\mathrm{r}}(\%)$ entre les deux valeurs est comparé à la précision fixée à $1 \%$ et suivant que $U_{\mathrm{s}}$ est plus grand ou plus petit que $U_{\mathrm{m}}$ on ajuste la borne supérieure ou inférieure de l'intervalle.

$$
E_{\mathrm{r}}(\%)=\frac{U_{\mathrm{m}}-U_{\mathrm{s}}}{U_{\mathrm{m}}} \times 100
$$

La procédure est répétée jusqu'à satisfaire le critère d'arrêt suivant la démarche décrite par l'algorithme de la figure 31. En appliquant la méthode semi-hertzienne, le modèle d'usure et la méthode d'identification, nous obtenons les résultats présentés figure 32 . Après identification, le coefficient d'usure $K^{\prime}$ vaut $1,5 \times 10^{-3}$, ce qui correspond à un coefficient d'usure d'Archard $K$ de $1,24 \times 10^{-4}$. En effet, la relation qui lit les deux coefficients est :

$$
K=\mu K^{\prime}
$$

Cette valeur est en accord avec les coefficients d'usure trouvés dans la bibliographie pour des contacts acier/acier.

\section{Prise en compte des dispersions dans la simulation}

\subsection{Approche probabiliste de la simulation de l'usure}

Pour prendre en compte les dispersions expérimentales du banc d'essai, l'approche probabiliste consiste à construire une densité de probabilité associée à chaque variable indépendante mal connue. Le principe du maximum d'entropie [32] ou encore le principe du maximum d'incertitude permet de construire la densité de probabilité $p_{X}(X)$ de la variable aléatoire $X$, à valeurs sur ]$-\infty,+\infty[$, en n'utilisant que la seule information disponible [33]. Ces informations sont des contraintes que doivent vérifier les densités de probabilité cherchées : le domaine de définition, la moyenne (moment d'ordre un), l'écart type (moment d'ordre deux) et des moments d'ordre supérieurs. La mesure de l'incertitude de la variable aléatoire $X$ est définie par l'entropie :

$$
S\left(p_{x}\right)=\int_{]-\infty,+\infty[} p_{x}(X) \log \left(p_{x}(X)\right) \mathrm{d} X
$$

Les $m$ informations connues $f_{m}$ sur la variable aléatoire sont définies par l'équation :

$$
E\left\{g_{i}(X)\right\}=\int_{]-\infty,+\infty[} g_{i}(X) p_{x}(X) \mathrm{d} X=f_{i}, i=1, \ldots, m
$$

La fonction de densité de probabilité est obtenue si elle maximise l'entropie $S\left(p_{X}\right)$ tout en vérifiant les $m$ contraintes et la condition de normalisation :

$$
\int_{]-\infty,+\infty[} p_{x}(X) \mathrm{d} X=1
$$

La forme de la densité de probabilité $p_{X}(X)$ est définie par l'équation :

$$
p_{x}(X)=\exp \left(-\lambda_{0}-\sum_{i=1}^{m} \lambda_{i} g_{i}(X)\right)
$$

Dans le cas du banc d'usure, nous avons vu que la variabilité des résultats d'usure est due à cinq paramètres expérimentaux incertains dont les informations connues sont :

- l'effort de contact $F:[0+\infty] ; m_{F}=340 \mathrm{~N} ; \sigma_{F}=33 \mathrm{~N}$

- le coefficient de frottement $\mu:[0+\infty] ; m_{\mu}=0,08$; $\sigma_{\mu}=0,02$

- le pseudoglissement longitudinal $v_{x}:[0+\infty] ; m_{v x}=$ $6 \times 10^{-2} \% ; \sigma_{v x}=4,6 \times 10^{-2} \%$

- le pseudoglissement de dérive $v_{y}$ : [-0,05 rad, $+0,05 \mathrm{rad}]$

- l'angle de $\operatorname{spin} \varphi_{x}:[-\infty+\infty] ; m_{\varphi x}=3,2 \times 10^{-3}$; $\sigma_{\varphi x}=1,4 \times 10^{-3}$ 

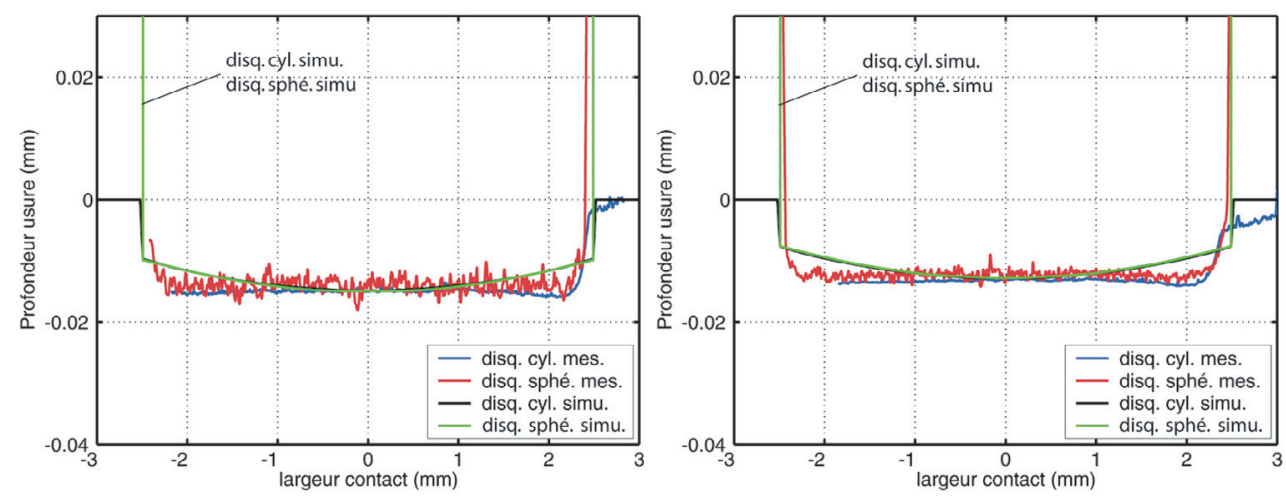

Fig. 32. Profils expérimentaux et profils simulés après identification.

Les résultats de minimisation pour les trois premiers paramètres sont récapitulés ci-dessous :

$$
\begin{aligned}
& F:\left|\begin{array}{l|l}
\lambda_{F 0}=50,6 \\
\lambda_{F 1}=-0,31 \\
\lambda_{F 2}=4,6 \times 10^{-4}
\end{array}, \quad \mu:\right| \begin{array}{l}
\lambda_{\mu 0}=6,4 \\
\lambda_{\mu 1}=-237,7 \\
\lambda_{\mu 2}=1,5 \times 10^{3}
\end{array}, \\
& v_{x}: \mid \begin{array}{l}
\lambda_{v_{x} 0}=-7,44 \\
\lambda_{v_{x} 1}=1,7 \times 10^{3} \\
\lambda_{v_{x} 2}=-1,7 \times 10^{3}
\end{array}, \quad \varphi_{x}: \begin{array}{l}
\lambda_{\varphi_{\mathrm{x}} 0}=2,6 \times 10^{5} \\
\lambda_{\varphi_{\mathrm{x}} 1}=-1,63 \times 10^{3} \\
\lambda_{\varphi_{\mathrm{x}} 2}=0,179
\end{array}
\end{aligned}
$$

Les densités de probabilité de chaque paramètre sont représentées sur la figure 33. Nous pouvons remarquer que les densités de probabilité de l'effort de contact et du coefficient de frottement sont en forme de « cloches » non symétriques; les valeurs moyennes de ces paramètres sont proches de la borne inférieure de chaque domaine de définition. Cette remarque est particulièrement vraie pour le pseudo glissement longitudinal pour lequel l'utilisation d'une distribution gaussienne aurait introduit une erreur particulièrement importante dans l'analyse de la dispersion.

La seule information disponible sur le pseudo glissement de dérive (qui provient d'un défaut angulaire de parallélisme des axes de rotation du galet par rapport au rondin) est le domaine de définition : [-0,02 rad +0,02 rad]. Dans ce cas, la densité de probabilité est uniforme et la fonction de répartition est une fonction linéaire.

Le principe de l'approche paramétrique est de définir un jeu de paramètres par tirage de Monte Carlo suivant les fonctions de densité de probabilité définies plus haut et de réaliser une simulation d'usure pour chaque tirage $i$. À partir d'un générateur aléatoire de nombres $\theta_{i}$ entre $[0,1]$ on obtient la réalisation $X_{i}$ associée par inversion de l'équation $\theta_{i}=F\left(X_{i}\right)$ où $F$ est la fonction de répartition (Fig. 34). La fonction de répartition associée à la variable aléatoire $X$ s'obtient par :

$$
F_{x}\left(p_{x}(X) \leq X_{\mathrm{D}}\right)=\int_{0}^{X_{\mathrm{D}}} \exp \left(-\lambda_{0}-\lambda_{1} X-\lambda_{2} X^{2}\right) \mathrm{d} X
$$

Pour s'assurer de la convergence de la méthode, au fur et à mesure des tirages on effectue les simulations d'usure et on trace l'évolution de la moyenne et de l'écart type de l'usure finale en fonction du nombre de réalisations. La stabilité de la solution de ces deux moments (moyenne et l'écart type) est atteinte au bout de 500 réalisations sur les 2100 réalisées.

\subsection{Analyse statistique des résultats}

Dans un premier temps, afin d'étudier la sensibilité de chaque paramètre sur la dispersion de l'usure, nous allons mettre en ouvre la méthode de Monte Carlo en ne faisant des tirages que sur un paramètre à la fois. Nous présentons cette sensibilité sous la forme d'intervalles de confiance IC de $90 \%$; c'est-à-dire d'intervalles qui englobent $90 \%$ des réalisations. La notion d'IC est illustrée sur la figure 35.

La définition de l'IC, déjà présentée dans [34], est spécifiée par les valeurs $X_{5} \%$ et $X_{95} \%$ qui sont respectivement le $5 \%$ quantile et le $95 \%$ quantile. L'intervalle de confiance peut-être défini pour n'importe quel pourcentage de confiance $(1-2 \alpha)$ avec les bornes $\alpha$ quantile et $(1-\alpha)$ quantile. Les $5 \%$ quantiles et les $95 \%$ quantiles de chaque paramètre sont définis comme suit :

$$
\begin{aligned}
& \left|\begin{array}{l}
F_{5 \%}=285,7 \mathrm{~N} \\
F_{95} \%=393,8 \mathrm{~N}
\end{array},\right| \begin{array}{l}
\mu_{5} \%=0,049 \\
\mu_{95} \%=0,0109
\end{array}, \mid \begin{array}{l}
v_{x 5} \%=3 \times 10^{-3} \% \\
v_{x 95} \%=0,0018 \%
\end{array}, \\
& \mid \begin{array}{l}
F_{y 5 \%}=|-0,0475|=0,0475 \\
F_{y 95 \%}=|0,0475|=0,0475
\end{array}, \begin{array}{l}
\varphi_{x 5} \%=8,7 \times 10^{-4} \mathrm{rad} \\
\varphi_{x 95} \%=5,6 \times 10^{-3} \mathrm{rad}
\end{array}
\end{aligned}
$$

Nous avons réalisé une simulation pour chaque valeur de quantile et tracé sur la figure 36 le résultat. Le paramètre qui donne la plus grande variabilité sur l'usure est clairement le pseudoglissement de dérive. Le pseudoglissement longitudinal et le pseudoglissement de spin ont également une influence non négligeable. Par contre, les variabilités sur l'effort de contact $F$ et le coefficient de frottement $\mu$ ont peu de répercussion sur l'usure par rapport au modèle moyen; ils pourraient être considérés comme déterministes.

Si on considère maintenant les tirages sur l'ensemble des paramètres dans les simulations, on obtient les résultats des réalisations $\theta_{i}$ regroupés sur la figure 37 . Nous constatons qu'avec l'approche paramétrique, l'usure 

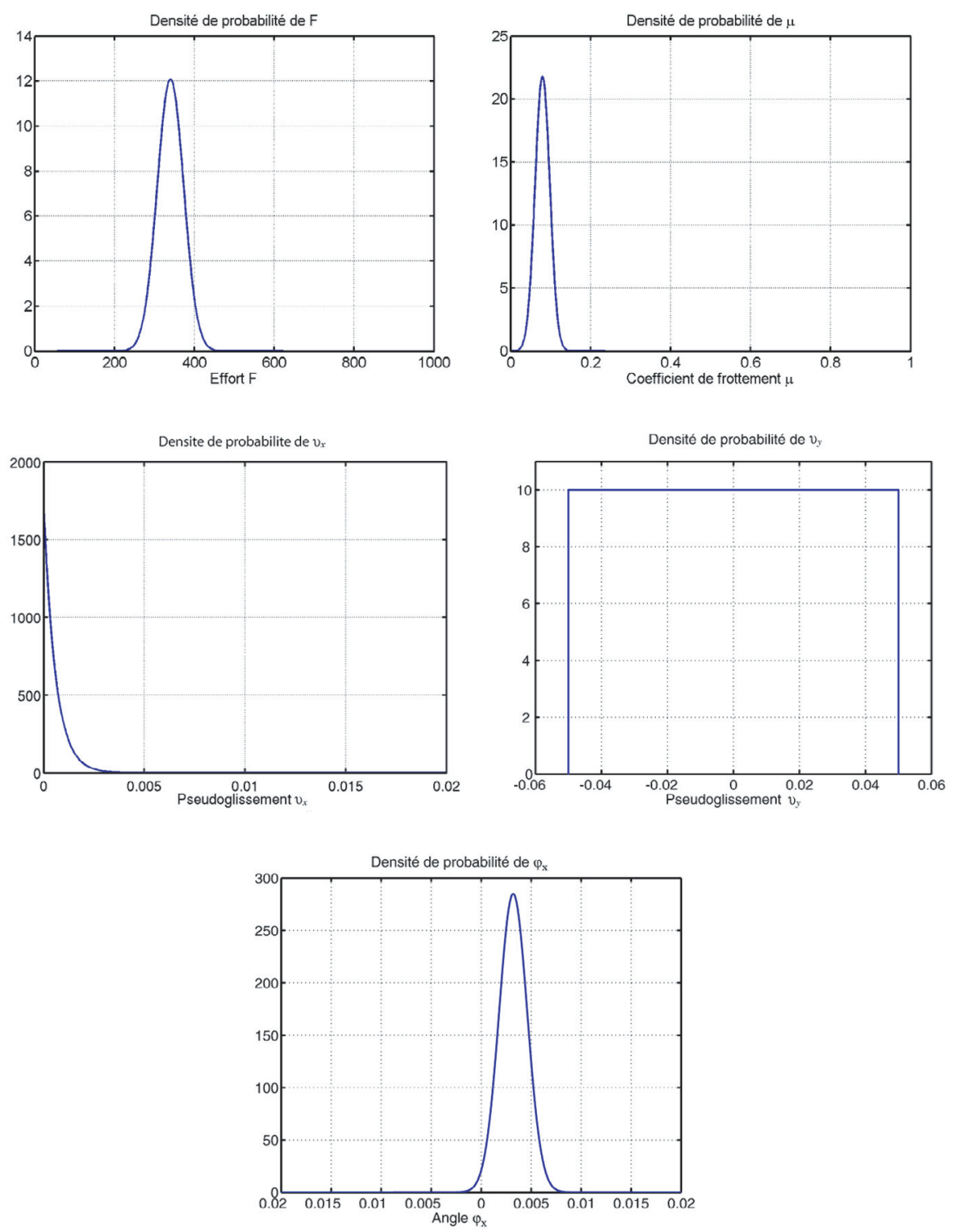

Fig. 33. Densités de probabilité calculées pour l'effort de contact, le coefficient de frottement, le pseudoglissement longitudinal, pseudoglissement de dérive et l'angle de spin.

maximale en fin de simulation $\left(1,32 \times 10^{6}\right.$ cycles $)$ varie de $0 \mathrm{~mm}$ à $0,165 \mathrm{~mm}$. Cette « fenêtre » englobe bien les essais d'usure réalisés avec le banc d'essai et illustre l'influence considérable des paramètres angulaires sur l'évolution de l'usure. À partir de la figure 37 et de la définition de l'intervalle de confiance à $90 \%$ nous pouvons définir les courbes enveloppes d'usure qui englobent $90 \%$ des simulations d'usure. Ce résultat permet de définir une fiabilité de $90 \%$ sur les courbes d'usure simulées. Ces courbes englobent les résultats expérimentaux des trois essais.

Les valeurs finales $\left(t=1,32 \times 10^{6}\right.$ cycles $)$ d'usure simulées présentent la statistique suivante :

$$
\left\{\begin{array}{l}
m_{U_{\max }}=0,063 \mathrm{~mm} \\
\sigma_{U_{\max }}=0,03 \mathrm{~mm}
\end{array}\right.
$$

La statistique donnée ci-dessus et le principe du maximum d'entropie, nous donnent accès à la fonction de densité de probabilité associée à l'usure maximale. En parallèle, les résultats de la figure 37 permettent de construire l'histogramme contenant les valeurs d'usure calculées pour 100 classes comprises entre 0 et 0,16 . La superposition de cet histogramme et de la densité de probabilité est présentée sur la figure 38. Nous pouvons constater que la distribution est non symétrique.

L'intérêt de notre modèle mécanique associé au modèle d'usure est que l'on peut comparer les profils simulés aux profils mesurés. La figure 39 compare les profils mesurés aux mêmes instants pour trois essais différents avec les profils simulés pour des IC de $90 \%$. Nous constatons que les profils simulés englobent bien les profils mesurés. L'essai 3 est le plus long : environ $3 \times 10^{6}$ cycles. Néanmoins les différents profils simulés englobent bien les profils expérimentaux. La modélisation de l'usure complétée par l'approche paramétrique permet 

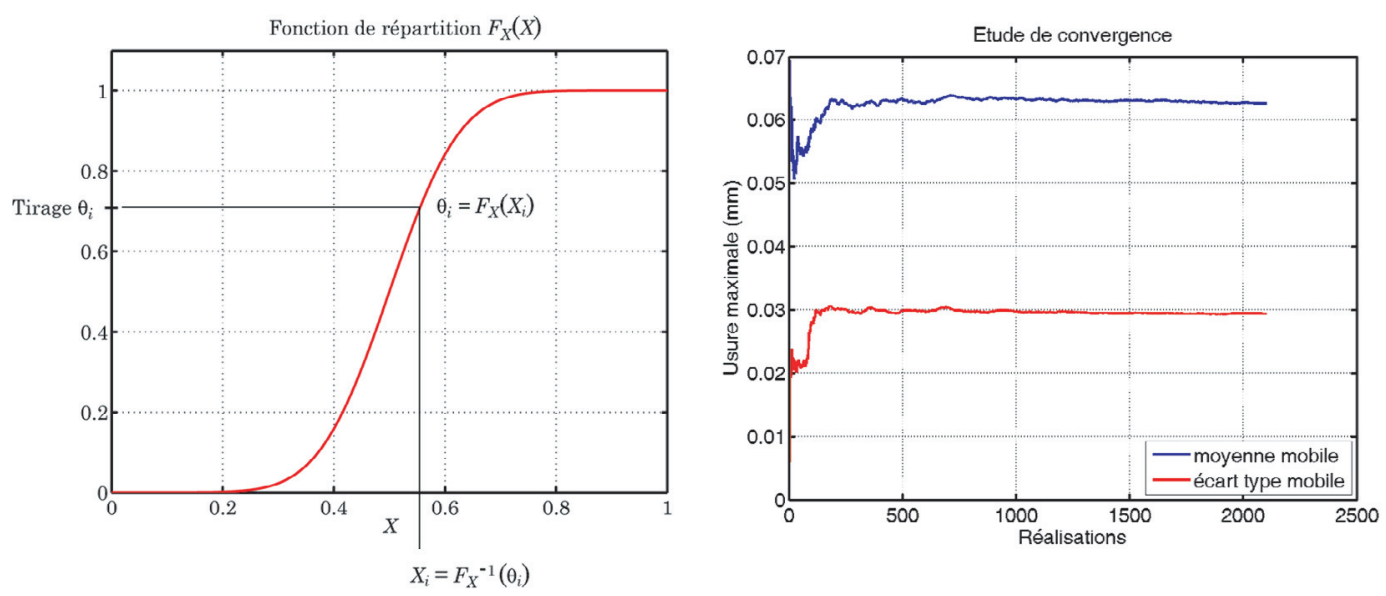

Fig. 34. Réalisation de tirages de Monte Carlo. Étude de convergence.

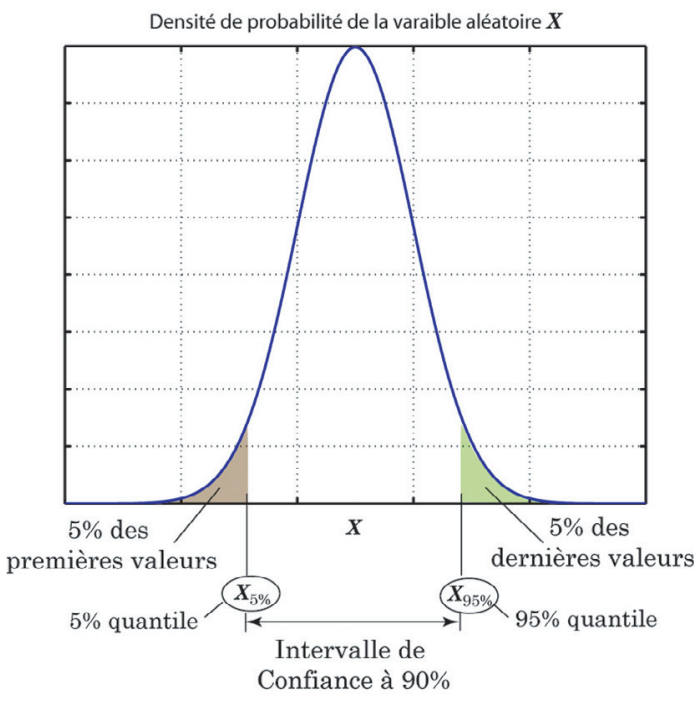

Fig. 35. Définition de l'intervalle de confiance.

de bien prendre en compte la variabilité observée sur les essais d'usure.

D'un point de vue industriel, la machine est déclarée « usée » lorsque l'usure maximale atteint l'usure critique $U_{\text {c. }}$. Afin de chiffrer la fiabilité de la machine, on étudie le pourcentage des réalisations de simulation qui atteignent $U_{\text {c }}$ et pour quelle durée cette valeur est atteinte. En construisant pour chaque niveau d'usure critique l'histogramme des pourcentages cumulés des réalisations de simulations qui atteignent le niveau d'usure défini par le critère d'usure, nous quantifions les durées de vie associées au $1 \%, 5 \%, 30 \%, 50 \%$ et $75 \%$ des réalisations. La figure 40 présente un exemple d'histogramme construit pour $U_{\mathrm{c}}=0,02 \mathrm{~mm}$.

L'usure critique de $0,02 \mathrm{~mm}$ est atteinte pour différents cycles d'essais. $10 \%$ des essais l'atteignent pour $0,12 \times 10^{6}$ cycles et $80 \%$ l'ont atteinte au bout de $0,55 \times 10^{6}$ cycles. 94,1\% des réalisations atteignent $U_{\mathrm{c}}$. En appliquant ce système de comptage sur chaque diagramme correspondant à un niveau d'usure critique, nous
Intervalle de confiance de $90 \%$ pour chaque variable probabiliste

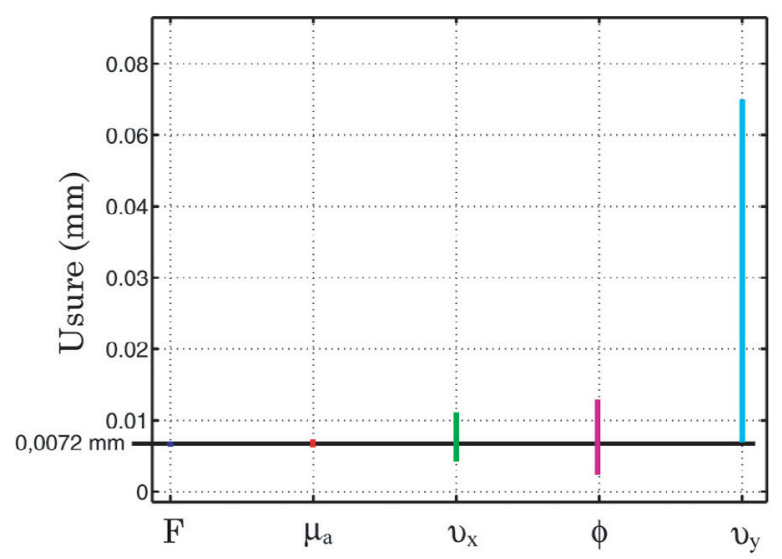

Fig. 36. Intervalle de confiance de $90 \%$ sur l'usure en fonction de la dispersion de chaque paramètre.

pouvons quantifier le pourcentage des réalisations qui vérifieront chaque critère d'usure (Fig. 41).

Sur la figure 41 nous constatons que toutes les réalisations n'atteignent pas la valeur faible d'usure maximale de $0,005 \mathrm{~mm}$. En effet $0,14 \%$ des réalisations n'atteignent jamais cette valeur. Ce graphique permet d'observer les proportions des réalisations qui atteignent les niveaux d'usure critique. Ainsi, il est possible de construire les pourcentages des réalisations qui atteignent les différents critères d'usure en fonction du temps d'essais à $1 \%, 5 \%, 30 \%, 50 \%$ et $75 \%$. Ces cinq courbes sont tracées sur la figure 42 .

Nous ajoutons des données fiabilistes (Fig. 42) aux résultats de simulation paramétrique de la figure 37 . La lecture des résultats fiabilistes s'effectue de la façon suivante : après avoir fixé un critère d'usure $(0,04 \mathrm{~mm}$ sur la Fig. 42), nous réalisons l'intersection des cinq courbes pour la valeur fixée d' $U_{\mathrm{C}}$. Pour l'exemple de la figure, nous constatons que $5 \%$ des réalisations atteignent la valeur critique de $0,04 \mathrm{~mm}$ pour $0,3 \times 10^{6}$ cycles, $50 \%$ 

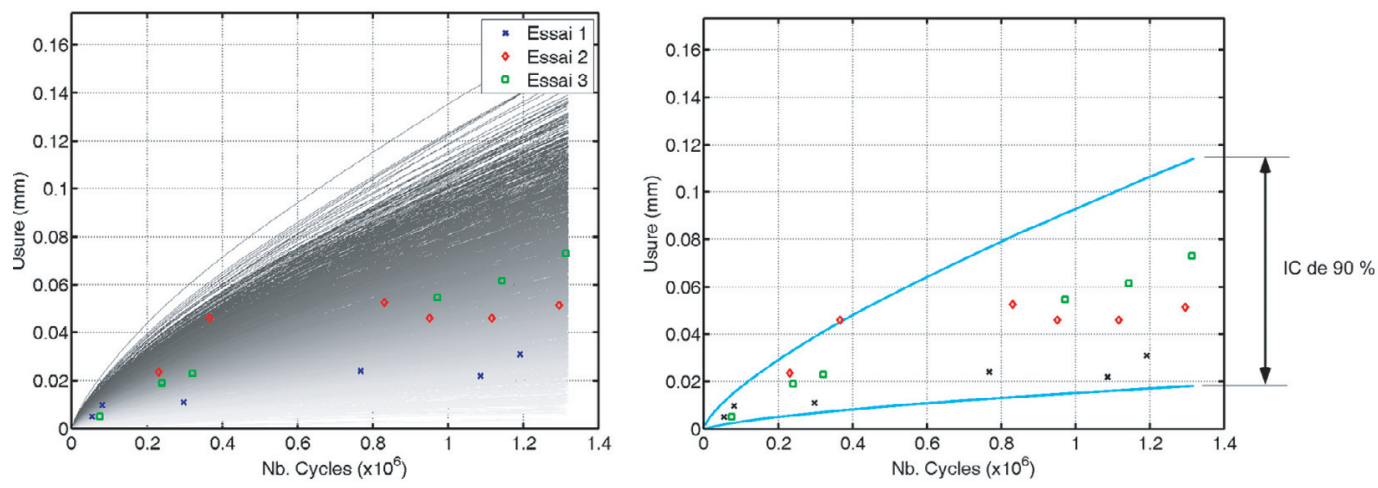

Fig. 37. Simulation probabiliste paramétrique de l'usure et résultats expérimentaux. Courbes enveloppes englobant $90 \%$ des simulations d'usure.

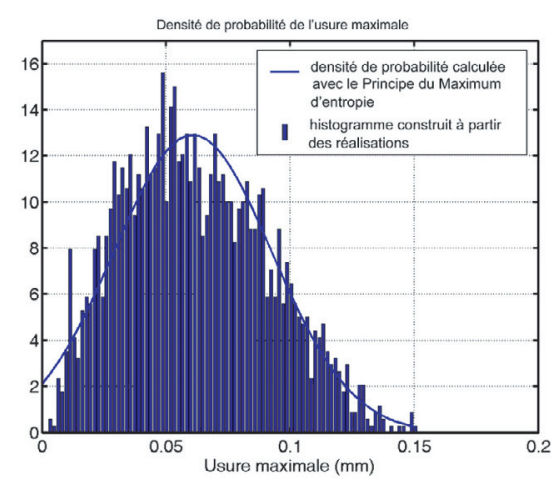

Fig. 38. Densité de probabilité de l'usure maximale.

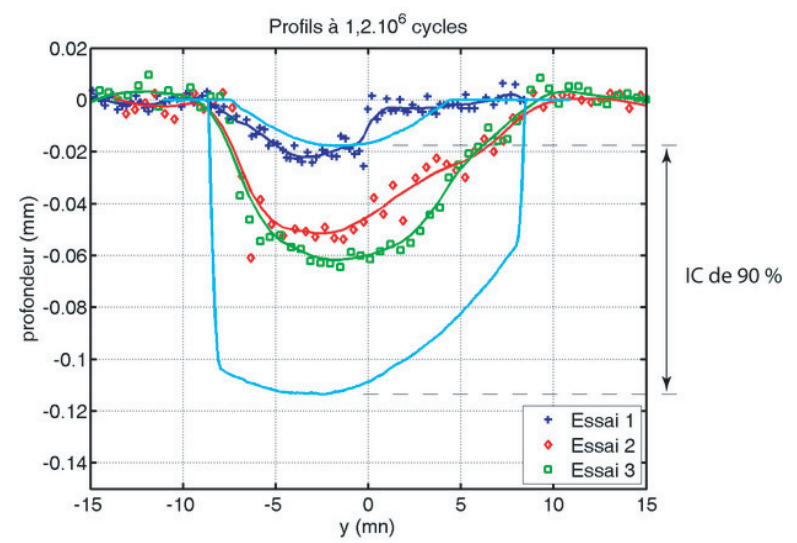

Fig. 39. Profils simulés, pour des IC de $90 \%$, et profils expérimentaux $1,2 \times 10^{6}$ cycles.

l'atteignent au bout de $0,75 \times 10^{6}$ cycles et $75 \%$ au bout de $1,235 \times 10^{6}$ cycles.

Industriellement, cela permet de définir quelles seront la durée de vie et la fiabilité des cames en fixant une usure critique à ne pas dépasser pour éviter les disfonctionnements des machines.

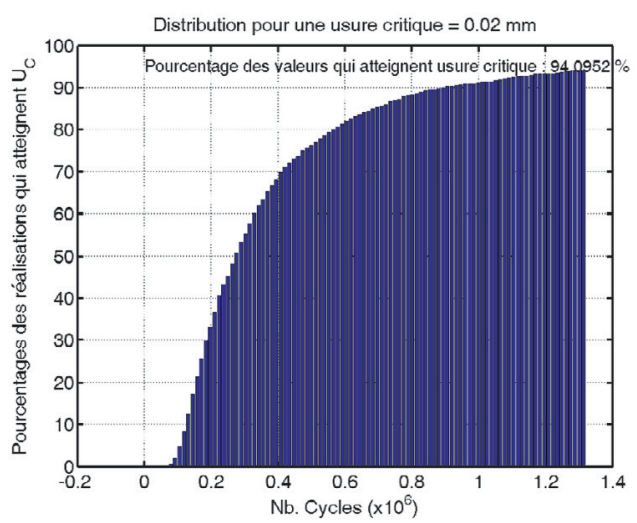

Fig. 40. Distribution cumulée des réalisations qui atteignent $U_{\mathrm{c}}=0,02 \mathrm{~mm}$

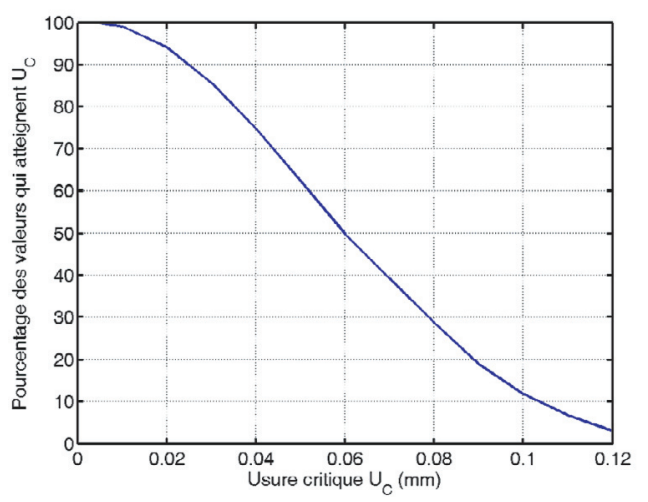

Fig. 41. Pourcentages des réalisations qui atteignent les différents critères d'usure.

\section{Conclusions}

L'objectif de l'étude présentée dans cet article était de « simuler rapidement l'évolution des profils d'usure en fonction du temps tout en prenant en compte les incertitudes expérimentales $»$.

Une approche simplifiée pour modéliser le contact roulant est proposée. Les résolutions du problème normal et du problème tangent, pour des conditions de contact 


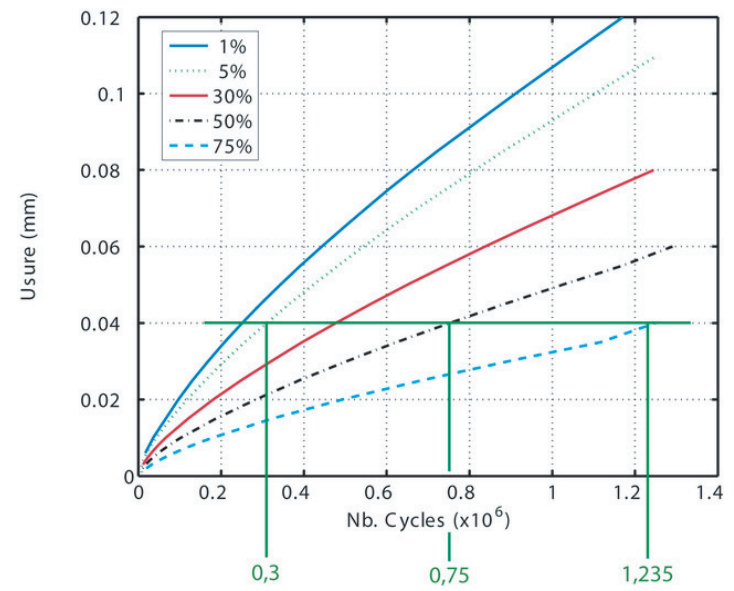

Fig. 42. Courbes à $1 \%, 5 \%, 30 \%, 50 \%$ et $75 \%$ des réalisations qui atteignent chaque critère d'usure.

non-hertziennes, sont validées en comparant la solution simplifiée à la solution de référence. La résolution du problème normal repose sur l'interpénétration des profils en contact à l'état non déformé et la raideur de contact par bande. Elle permet de bien approximer la surface de contact ainsi que la pression de contact si l'on opère une correction sur les courbes et une diffusion entre les bandes de contact. L'usure dépend de la puissance dissipée linéique et la résolution du problème tangent, basée sur l'algorithme FASTSIM, donne une bonne approximation de cette puissance. La résolution du problème de contact par l'approche simplifiée est 1700 fois plus rapide que la solution exacte ce qui rend la simulation exploitable dans un environnement de type bureau d'étude. De plus cela a permis de réaliser les simulations de l'approche probabiliste paramétrique dans un temps réduit.

Le modèle d'usure développé permet de bien simuler l'évolution du profil du rondin due à l'usure. L'usure est générée localement pour $N$ passages de galet ce qui permet d'accélérer la simulation sans altérer le résultat. Cette simulation de l'usure reproduit bien qualitativement les évolutions observées expérimentalement. Passée une phase d'usure où les surfaces s'accommodent jusqu'à la conformité des solides en contact, la puissance dissipée est uniforme ce qui rend l'évolution de l'usure maximale linéaire en fonction du temps.

L'approche probabiliste paramétrique propose une méthode pour «quantifier» la dispersion de l'usure. L'utilisation du principe du maximum d'entropie pour calculer les densités de probabilité associées à chaque paramètres présente deux avantages : il est simple à mettre en œuvre numériquement et il permet de définir la pdf sur un intervalle de définition fini. Nous montrons qu'en tenant compte des dispersions de chaque paramètre les réalisations de simulation d'usure englobent les essais d'usure. La dispersion sur l'usure maximale est due en grande partie à la dispersion sur les paramètres de glissement et la réalisation d'essais d'usure comparatifs nécessite de bien contrôler les pseudoglissements longitudinaux, transversaux et de spin.
Remerciements. Au LISMMA de SupMéca à Saint-Ouen pour nous avoir permis de réaliser nos essais bi-disques sur leur tribomètre.

\section{Références}

[1] L. Chevalier, S. Cloupet, M. Quillien, Friction and wear during twin-disc experiments under ambient and cryogenic conditions, Trib. Inter. (2006) 1376-1387

[2] L. Chevalier, H. Chollet, Endommagement des pistes de roulement, Mécanique \& Industries 1 (2000) 77-103

[3] M. Quillien, R. Gras, L. Collongeat, Th. Kachler, A testing device for rolling-sliding behavior in harsh environments: the twin-disk cryotribometer, Trib. Int. 34 (2001) 287-292

[4] T. Ohyama, H. Maruyama, Traction and slip at higher rolling speeds: some experiments under dry friction and water lubrification, J. Japan Soc. Lubrication Eng. 21 (1976) 434-440

[5] H. Chollet, Etude en similitude mécanique des efforts tangents au contact roue-rail, Thèse Paris-6, 1991

[6] N. Fillot, I. Iordanoff, Y. Berthier, Simulation of wear through mass balance in dry contact, J. Trib. 127 (2005) 973-981

[7] N. Fillot, I. Iordanoff, Y. Berthier, Modeling and the third body concept, Wear 262 (2007) 949-957

[8] N. Fillot, I. Iordanoff, Y. Berthier, Modelling third body flows with a discrete element method, a tool for understanding wear with adhesive particles, Trib. Int. 40 (2007) 973-981

[9] J.F. Archard, Contact and rubbing of flat surfaces, J. Appl. Phys. 24 (1953) 981-988

[10] T. Jendel, Prediction of wheel profile wear-comparisons with field measurements, Wear 253 (2002) 89-99

[11] M.A. Moore, Abrasive wear, Materials in engenering applications 1 (1978) 97-111

[12] Zi-Li Li, J.J Kalker, Simulation of Severe Wheel-Rail Wear, Proceedings International Conference Computers in Railways, ed. WITPRESS, Southampton UK, 1998, 6, pp. 393-402.

[13] P. Põdra, S. Anderson, Wear calculations with the Winkler surface model, Wear 207 (1997) 79-85

[14] I.G. Goryacheva, P.T. Rajeev, T.N. Farris, Wear in Partial Slip Contact, J. Tribology 123 (2001) 848-856

[15] U. Olofsson, T. Telliskivi, Plastic deformation and friction of two rail steels, a full-scale test and a laboratory study, Wear 254 (2003) 80-93

[16] T. Telliskivi, Simulation of wear in a rolling-sliding contact by a semi-Winkler model and the Archard's wear law, Wear 256 (2004) 817-831

[17] R. Enblom, M. Berg, Simulation of railway wheel profile development due to wear, influence of disc braking and contact environment, Wear 258 (2005) 1055-1063

[18] N.H. Kim, D. Won, D. Burris, B. Holtkamp, G.R. Gessel, P. Swanson, W.G. Sawyer, Finite element analysis experiments of metal/metal wear in oscillatory contacts, Wear 258 (2005) 1787-1793

[19] J. Boussinesq, Application des potentiels à l'équilibre et du mouvement des solides élastiques, ed. GauthierVillars, Paris, 1885 
[20] P.J. Vermeulen, K.L. Johnson, Contact of non-spherical elastic bodies transmitting tangential forces, Trans. ASME J. Appl. Mech. 31 (1964) 338-340

[21] E. Legrand, F. Robbe-Valloire, Analyse des efforts tangentiels dans les contacts billes-bagues non lubrifiés, Revue Française de Mécanique, Bulletin S.F.M. (1994) 93-102

[22] F.W. Carter, On the action of a locomotive driving wheel, Proc. Royal Soc. London A112 (1926) 151-157

[23] J.J. Kalker, The computation of three-dimensional rolling contact with dry friction, Int. J. Numer. Methods Eng. 14 (1979) 1293-1307

[24] J.J. Kalker, Wheel/rail wear calculations with the program CONTACT, Contact Mechanics and Wear of Rail/Wheel System 2 (1987) 3-26

[25] J.J. Kalker, A Fast Algorithm for the Simplified Theory of Rolling Contact, Vehicle Syst. Dyn. 11 (1982) 1-13

[26] B. Jacobson, J.J. Kalker, Rolling Contact Phenomena: Linear Elasticity, CISM Lecture No. 411, Springer, Berlin, 2000

[27] J. B. Ayasse, H.Chollet, Determination of the Wheel Rail Contact Patch in Semi-Hertzian Conditions, Veh. Syst. Dyn. 43 (2005) 161-172 (2005)

[28] W. Kik, J. Piotrowski, A fast approximative method to calculate normal load at contact between wheel and rail, and creep forces during rolling, Proceedings of the 2nd mini-conference on contact mechanics and wear of rail/wheel systems, Budapest, Technical University of Budapest, 29-31 July, 1996, pp. 52-61

[29] L. Chevalier, S. Cloupet, A. Eddhahak, Contributions à la modélisation simplifiée de la Mécanique des contacts roulants, Mécanique \& Industries 7 (2006) 155-168

[30] X. Quost, M. Sebes, A. Eddhahak-Ouni, J.B. Ayasse, H. Chollet, P.E. Gautier, F. Thouverez, Assessment of a semi-Hertzian method for determination of wheel-rail contact patch, Vehicle System Dynamics 44 (2006) 789814

[31] B. Nayroles, G. Touzot, P. Villon, La méthode des éléments diffus, C. R. Acad. Sci. Paris, Ser. II 313 (1991) $133-138$

[32] E.T. Jaynes, Information theory and statistical mechanics, Phys. Rev. 106 (1957) 620-630 ; 108 (1957) 171-190

[33] J.N. Kapur, H.K. Kesavan, Entropy optimisation principle with applications, ed. Academic Press, San Diego, 1992

[34] L. Chevalier, S. Cloupet, C. Soize, Probabilistic approach for wear modelling in steady state rolling contact, Wear 258 (2005) 1543-1554 